\title{
Opérateurs différentiels associés à certaines représentations unitaires d'un groupe de Lie résoluble exponentiel
}

(Differential Operators Associated with Certain Unitary Representations of a Lie Resolvable Exponential Group)

ALI BAKLOUTI ${ }^{1}$ and HIDÉNORI FUJIWARA ${ }^{2}$

${ }^{1}$ Faculté des Sciences de Sfax, Département de Mathématiques, Route de Soukra, 3038 Sfax, Tunisie.e-mail: ali.baklouti@fss.rnu.tn

${ }^{2}$ Faculté de Technologie à Kyushu, Université de Kinki, Iizuka 820, Japon.

e-mail:fujiwara@fuk.kindai.ac.jp

(Received: 11 November 2001; accepted in final form 5 August 2002)

Résumé. Soient $G=\exp g$ un groupe de Lie résoluble exponentiel et $H=\exp \mathfrak{h}$ un sousgroupe connexe de $G$. Soient $\chi$ un caractère unitaire de $H$ et $\tau=\operatorname{Ind}_{H}^{G} \chi$. Soit $D_{\tau}(G / H)$ l'algèbre des opérateurs différentiels $G$-invariants sur $G / H$. Une question posée par Duflo et CorwinGreenleaf consiste à voir si la finitude des multiplicités de $\tau$ est équivalente à la commutativité de $D_{\tau}(G / H)$. Nous répondons positivement à cette question quand $H$ est normal dans $G$. Lorsque $H$ n'est pas normal, nous préparons le terrain pour d'espaces homogènes nilpotents et nous répondons à la question dans différents cas. Nous étudions finalement l'algèbre $D_{\pi}(G)^{H}, \pi \in \hat{G}$ des opérateurs différentiels qui laissent l'espace des vecteurs $C^{\infty}$ de $\pi$ invariant et qui commuttent avec l'action de $H$ sur cet espace.

Mathematics Subject Classification (2000). 22E27.

Mots clés. désintégration, groupe de Lie exponentiel, multiplicité, orbite, polarisation.

\section{Introduction}

Soit $G=\exp g$ un groupe de Lie réel, connexe, simplement connexe et résoluble exponentiel. Soit $\mathfrak{g}$ l'algèbre de Lie de $G$. Alors l'application exponentielle:

exp: $\mathfrak{g} \longrightarrow G$

est un difféomorphisme de $\mathfrak{g}$ sur $G$. Soit $\mathfrak{g}^{\star}$ l'espace vectoriel dual de $\mathfrak{g}$. $G$ agit $\operatorname{sur} \mathfrak{g}^{\star}$ par la représentation coadjointe. Etant donnés un sous-groupe analytique $H=\exp \mathfrak{h}$ et un caractère unitaire $\chi$ sur $H$, on construit la représentation monomiale (induite) $\tau=\operatorname{Ind}_{H}^{G} \chi$. Précisons notre façon de construire la représentation monomiale.

Soient $\mathrm{d} g$ une mesure de Haar à gauche sur $G$ et $\Delta_{G}$ la fonction module de $G$ de sorte que l'on a:

$$
\int_{G} F\left(g x^{-1}\right) \mathrm{d} g=\Delta_{G}(x) \int_{G} F(g) \mathrm{d} g \quad(x \in G)
$$


pour toute fonction $F$ appartenant à l'espace $K(G)$ des fonctions continues sur $G$ à support compact. Il est bien connu que:

$$
\Delta_{G}(x)=|\operatorname{det} \operatorname{Ad} x|^{-1} \quad(x \in G) .
$$

On note $\Delta_{H, G}$ le caractère de $H$ à valeurs dans $\mathbf{R}_{+}$défini par:

$$
\Delta_{H, G}(h)=\frac{\Delta_{H}(h)}{\Delta_{G}(h)} .
$$

On a, pour $X$ dans $\mathfrak{h}$,

$$
\Delta_{H, G}(\exp X)=\exp \left(\operatorname{Tr}_{\mathfrak{g} / \mathfrak{h}} X\right) .
$$

Soit $K(G, H)$ l'espace des fonctions numériques $F$ continues sur $G$, à support compact modulo $H$ et qui vérifient:

$$
F(g h)=\Delta_{H, G}(h) F(g) \quad(g \in G, h \in H) .
$$

$G$ agit sur cet espace par translation à gauche. On sait ([5]) que, à un scalaire multiplicatif près, il existe une et une seule forme linéaire positive $G$-invariante sur $K(G, H)$. On la note $v_{G, H}$ et on l'écrit sous la forme d'une intégrale:

$$
v_{G, H}(F)=\oint_{G / H} F(g) \mathrm{d} v(g)
$$

Si $\Delta_{H}=\Delta_{G}$ sur $H, v_{G, H}$ est une mesure $G$-invariante sur l'espace homogène $G / H$.

On note $K(f, \mathfrak{h}, G)$ l'espace des fonctions numériques $F$ continues sur $G$ qui vérifient:

$$
F(g h)=\Delta_{H, G}^{1 / 2}(h) \chi(h)^{-1} F(g) \quad(g \in G, h \in H)
$$

et dont le support est compact modulo $H$. Si $F$ appartient à $K(f, \mathfrak{h}, G)$, la fonction $g \mapsto|F(g)|^{2}$ appartient à $K(G, H)$ et l'on pose:

$$
\|F\|^{2}=\oint_{G / H}|F(g)|^{2} \mathrm{~d} v(g) .
$$

La représentation induite $\tau=\operatorname{Ind}_{H}^{G} \chi$ de $G$ se réalise par translation à gauche dans l'espace de Hilbert $\mathcal{H}_{\tau}$ complété de $K(f, \mathfrak{h}, G)$ muni de la norme définie ci-dessus.

Il existe $f \in \mathfrak{g}^{\star}$ telle que $\chi$ s'écrive $\chi_{f}(\exp X)=\mathrm{e}^{\mathrm{if}(X)}(X \in \mathfrak{h})$. Dans cette situation $\chi$ se notera éventuellement $\chi_{f}$. C'est le sous-espace affine $\Gamma_{\tau}=f+\mathfrak{h}^{\perp}, \mathfrak{h}^{\perp}$ désignant l'annihilateur de $\mathfrak{h}$ dans $\mathfrak{g}^{\star}$ qui joue un rôle fondamental pour étudier $\tau$. Soit $\tilde{\mu}$ une mesure positive finie sur $\mathfrak{g}^{\star}$ équivalente à la mesure de Lebesgue sur $\Gamma_{\tau}$. Nous prenons l'image $\mu$ de $\tilde{\mu}$ par l'application de Kirillov-Bernat $\Theta: \mathrm{g}^{\star} \rightarrow \hat{G}$, le dual unitaire de $G$. $\hat{G}$ se réalise comme l'espace des orbites coadjointes $\mathfrak{g}^{\star} / G$ au moyen de la bijection induite $\bar{\Theta}: \mathrm{g}^{\star} / G \rightarrow \hat{G}$ (cf. [5, 22]). Pour $\pi \in \hat{G}$ on note $\Omega(\pi)=\Omega_{G}(\pi)=$ $\Theta^{-1}(\pi)$ l'orbite associée. La désintégration centrale canonique de $\tau$ s'écrit [14]

$$
\tau \simeq \int_{\hat{G}}^{\oplus} m(\pi) \pi \mathrm{d} \mu(\pi)
$$


avec la fonction de multiplicités $m(\pi)$ donnée par le nombre des $H$-orbites contenues dans $\Gamma_{\tau} \cap \Omega(\pi)$.

Pour $l \in \mathfrak{g}^{\star}$, on note $B_{l}$ la forme bilinéaire antisymétrique sur $\mathfrak{g}$ donnée par

$$
B_{l}(X, Y)=l([X, Y]) \text {. }
$$

Il est bien connu que les deux conditions suivantes sont équivalentes (cf. [23]):

1. $\tau$ est à multiplicités finies

2. $\mathfrak{h}+\mathfrak{g}(l)$ est génériquement lagrangien dans $\mathfrak{g}$ relativement à la forme $B_{l}$.

Pour une représentation unitaire $\rho$ de $G, \mathcal{H}_{\rho}$ dénote l'espace de Hilbert de $\rho, \mathcal{H}_{\rho}^{+\infty}$ l'espace des vecteurs $C^{\infty}$ de $\rho$ muni de sa topologie usuelle [26] et $\mathcal{H}_{\rho}^{-\infty}$ l'antidual de $\mathcal{H}_{\rho}^{+\infty}$. Pour $a \in \mathcal{H}_{\rho}^{ \pm \infty}$ et $b \in \mathcal{H}_{\rho}^{\mp \infty}$, on note $\langle a, b\rangle$ l'image de $b$ par $a$. Alors l'espace $\mathcal{H}_{\rho}^{+\infty}$ est un sous-espace invariant par l'action de $\rho$. De même, $G$ agit sur $\mathcal{H}_{\rho}^{-\infty}$ de la façon suivante:

$$
\langle\rho(g) a, b\rangle=\left\langle a, \rho\left(g^{-1}\right) b\right\rangle, \quad a \in \mathcal{H}_{\rho}^{-\infty}, b \in \mathcal{H}_{\rho}^{+\infty}, g \in G .
$$

Si $c$ est un caractère complexe d'un sous-groupe $K$ de $G$, on pose

$$
\left(\mathcal{H}_{\rho}^{-\infty}\right)^{K, c}=\left\{a \in \mathcal{H}_{\rho}^{-\infty}: \rho(k) a=c(k) a, \quad \forall k \in K\right\} .
$$

En revenant à notre représentation monomiale $\tau$ et en désignant par $e$ l'élément neutre de $G$, nous considérons la mesure de Dirac:

$$
\delta_{\tau}: \mathcal{H}_{\tau}^{+\infty} \ni \psi \mapsto \overline{\psi(e)} .
$$

Il s'ensuit que $\delta_{\tau}$ appartient à $\left(\mathcal{H}_{\tau}^{-\infty}\right)^{H, c}$ avec $c=\chi \Delta_{H, G}^{1 / 2}$. Alors, suivant la désintégration de $\tau, \delta_{\tau}$ elle-même se désintègre. En supposant, par exemple, que $m(\pi)\langle\infty$ $\mu$-presque partout, on a

$$
\delta_{\tau}=\int_{\hat{G}}^{\oplus}\left(\sum_{k=1}^{m(\pi)} a_{\pi}^{k}\right) \mathrm{d} \mu(\pi)
$$

avec certains $a_{\pi}^{k} \in\left(\mathcal{H}_{\pi}^{-\infty}\right)^{H, c}$ ([25]). Cette désintégration s'appelle la formule de Plancherel pour $\tau$. Formons pour $\varphi \in C_{c}^{\infty}(G)$ un élément $\varphi^{\chi}$ de $\mathcal{H}_{\tau}^{+\infty}$ par la formule

$$
\varphi^{\chi}(g)=\int_{H} \varphi(g h) \chi(h) \Delta_{H, G}^{-1 / 2}(h) \mathrm{d} h, \quad(g \in G)
$$

avec une mesure de Haar à gauche $d h$ sur $H$. La formule de Plancherel pour $\tau$ se récrit alors, pour toute $\varphi \in C_{c}^{\infty}(G)$,

$$
\varphi^{\chi}(g)=\int_{\hat{G}}\left(\sum_{k=1}^{m(\pi)}\left\langle\pi\left(\varphi_{g}\right) a_{\pi}^{k}, a_{\pi}^{k}\right\rangle\right) \mathrm{d} \mu(\pi)
$$

où $\varphi_{g}$ s'obtient par $\varphi_{g}(x)=\varphi(g x), x \in G$.

Considérons maintenant une représentation unitaire et irréductible $\pi$ associée à une orbite coadjointe $\Omega(\pi) \subset \mathrm{g}^{\star}$ d'une forme linéaire $f$. Soit $H$ un sous-groupe analytique de $G$ d'algèbre de Lie $\mathfrak{h}$ et considérons la restriction $\pi_{\mid H}$ de $\pi$ à $H$. Soit $\mu_{\pi}$ une 
mesure finie sur $\Omega(\pi)$ qui est équivalente à la mesure $G$-invariante. L'application de Kirillov $\theta_{H}: \mathfrak{h}^{\star} \longrightarrow \hat{H}$ induit un isomorphisme de la structure borélienne de l'espace des orbites $\mathfrak{h}^{\star} / H$ vers $\hat{H}$. On considère la mesure $\eta=\eta_{H}^{\pi}=\left(\theta_{H} \circ p\right) \star\left(\mu_{\pi}\right)$, qui est l'image de $\mu_{\pi}$ via l'application $\theta_{H} \circ p: \mathfrak{g}^{\star} \longrightarrow \hat{H}$ où $p: \mathfrak{g}^{\star} \rightarrow \mathfrak{h}^{\star}$ est la projection canonique. Pour $\sigma \in \hat{H}$, soit $n_{\pi}(\sigma)$ le nombre de $H$-orbites contenues dans $\Gamma(\pi, \sigma)=$ $\Omega_{G}(\pi) \cap p^{-1}\left(\Omega_{H}(\sigma)\right)$. Alors d'après H. Fujiwara (cf. [12])

$$
\pi_{\mid H} \simeq \int_{\hat{H}}^{\oplus} n_{\pi}(\sigma) \sigma \mathrm{d} \eta(\sigma)
$$

Soit maintenant $N$ un groupe de Lie nilpotent, connexe et simplement connexe. On considère une représentation unipotente de $N$ sur un espace vectoriel réel $V$ de dimension finie. Soit $v \in V$ un vecteur invariant par l'action de $N$, i.e. $n \cdot v=v$ pour tout $n \in N$. Posons pour $x \in V$ arbitairement fixé, $L_{x}=\{x+t v, t \in \mathbf{R}\}$, la ligne droite passant par $x$ et ayant la direction de $v$. Alors, il $y$ a deux possibilités: ou bien $L_{x} \cap N \cdot x=L_{x}$, ou bien $L_{x} \cap N \cdot x=\{x\}$. Conformément à ces deux possibilités, nous dirons que l'orbite $N \cdot x$ est ou bien saturée ou bien non-saturée dans la direction de $\mathbf{R} v$. Nous utiliserons dans toute la suite ce fait appliqué à la représentation coadjointe, où le vecteur invariant $v$ sera une forme linéaire qui s'annule sur un idéal $\mathrm{g}_{0}$ de codimension $1 \mathrm{de} \mathrm{g}$. Dans cette situation, nous dirons que l'orbite en question est ou bien saturée ou bien non-saturée par rapport à $\mathfrak{g}_{0}$.

\section{Description Des Résultats}

Cet article est consacré à l'étude des opérateurs différentiels qui sont associés à des représentations unitaires d'un groupe de Lie résoluble exponentiel $G$. Ces représentations sont précisément des induites à partir d'un sous-groupe analytique d'un caractère unitaire on bien des restrictions aux sous-groupes analytiques d'une représentation unitaire et irréductible de $G$. C'est alors la méthode des orbites qui se révèle très utile pour notre étude.

Soit $\tau=\operatorname{Ind}_{H}^{G} \chi_{f}$ la représentation monomiale définie comme ci-dessus. On définit une certaine algèbre des opérateurs différentiels $G$-invariants associée à cette représentation. On considère l'algèbre $\operatorname{Diff}(G)$ des opérateurs différentiels qui sont $C^{\infty}$ et qui laisse $C^{\infty}(G, \tau)=C^{\infty}(G) \cap K(f, \mathfrak{h}, G)$ stable. Notre algèbre $D_{\tau}(G / H)$ est l'ensemble des restrictions $D \mid C^{\infty}(G, \tau)$ de $D \in \operatorname{Diff}(G)$ commutant avec les translations à gauche de $G \operatorname{sur} C^{\infty}(G, \tau)$.

Décrivons l'algèbre $D_{\tau}(G / H)$ en termes de l'algèbre enveloppante $\mathcal{U}(\mathfrak{g})$ de $\mathfrak{g}_{\mathrm{C}}$ (cf. [7, 10, 28]). Pour $g \in G$ ou $A \in \mathcal{U}(\mathrm{g})$, on note $R_{g}$ ou $R(A)$ (resp. $L_{g}$ ou $\left.L(A) z\right)$ l'action à droite (resp. à gauche). Soient $\mathcal{U}(\mathfrak{g}, \tau)$ l'ensemble des $A \in \mathcal{U}(\mathfrak{g})$ tels que $R(A)$ laisse $C^{\infty}(G, \tau)$ stable, $\mathfrak{a}_{\tau}$ le sous-espace vectoriel de $\mathcal{U}(\mathfrak{g})$ engendré par $Y+i f(Y)-\frac{1}{2} \operatorname{Tr} \operatorname{ad}_{\mathfrak{g} / \mathfrak{h}} Y, Y$ parcourant $\mathfrak{h}$, et $\mathcal{U}(\mathfrak{g}) \mathfrak{a}_{\tau}$ l'idéal gauche engendré par $\mathfrak{a}_{\tau}$. Alors il se trouve que

$$
\mathcal{U}(\mathfrak{g}, \tau)=\left\{A \in \mathcal{U}(\mathfrak{g}):[A, \mathfrak{h}] \subset \mathcal{U}(\mathfrak{g}) \mathfrak{a}_{\tau}\right\}
$$

et que l'application $A \longmapsto R(A) \mid C^{\infty}(G, \tau)$ nous donne un homomorphisme $\operatorname{de} \mathcal{U}(\mathrm{g}, \tau)$ 
sur $D_{\tau}(G / H)$ avec le noyau $\mathcal{U}(\mathfrak{g}) \mathfrak{a}_{\tau}$. En somme,

$$
D_{\tau}(G / H) \simeq \mathcal{U}(\mathfrak{g}, \tau) / \mathcal{U}(\mathfrak{g}) \mathfrak{a}_{\tau}=\left(\mathcal{U}(\mathfrak{g}) / \mathcal{U}(\mathfrak{g}) \mathfrak{a}_{\tau}\right)^{H},
$$

où le dernier représente l'algèbre des éléments $H$-invariants de $\mathcal{U}(\mathrm{g}) / \mathcal{U}(\mathrm{g}) \mathfrak{a}_{\tau}$.

De plus, si le couple $(\mathfrak{g}, \mathfrak{h})$ est réductif dans le sens qu'il existe un supplémentaire $\mathfrak{m}$ de $\mathfrak{h}$ dans $\mathfrak{g}$ tel que $[\mathfrak{h}, \mathfrak{m}] \subset \mathfrak{m}$, on voit que

$$
\mathcal{U}(\mathfrak{g}, \tau)=\mathcal{U}^{H}(\mathfrak{g})+\mathcal{U}(\mathfrak{g}) \mathfrak{a}_{\tau}
$$

$\operatorname{avec} \mathcal{U}^{H}(\mathfrak{g})=\{A \in \mathcal{U}(\mathfrak{g}):[A, Y]=0, \forall Y \in \mathfrak{h}\}$. D'où

$$
D_{\tau}(G / H) \simeq \mathcal{U}^{H}(\mathfrak{g}) / \mathcal{U}^{H}(\mathfrak{g}) \cap \mathcal{U}(\mathfrak{g}) \mathfrak{a}_{\tau} .
$$

On se demande: quand est-ce que $D_{\tau}(G / H)$ est commutative $([7,11])$ ? Dans leur travail fondamental [7], L. Corwin et F. Greenleaf ont démontré que $D_{\tau}(G / H)$ est commutative si $G$ est nilpotent et si $m(\pi)<\infty \mu$-presque partout (cf. [3, 18, 19]). Dans bien des cas connus où $m(\pi)<\infty \mu$-presque partout (cf. [1, 3, 4, 8, 15]), l'algèbre $D_{\tau}(G / H)$ est isomorphe à l'algèbre $\mathrm{C}\left[\Gamma_{\tau}\right]^{H}$ des fonctions polynomiales $H$-invariantes sur $\Gamma_{\tau}$. Ce qui nous amène à la conjecture suivante:

CONJECTURE 2.1 (cf. [7, 8]). Sous les hypothèses que G est nilpotent et que les multiplicités de $\tau$ sont finies presque partout, l'algèbre $D_{\tau}(G / H)$ est isomorphe à l'algèbre des fonctions polynomiales $H$-invariantes sur l'espace affine $f+\mathfrak{h}^{\perp}$.

En effet, lorsqu'on a la formule de Plancherel (1) pour $\tau$, un calcul direct [16] nous donne

$$
\left(R(A) \varphi^{\chi}\right)(g)=\int_{\hat{G}}\left(\sum_{k=1}^{m(\pi)}\left\langle\pi\left(\varphi_{g}\right) a_{\pi}^{k}, \pi(\bar{A}) a_{\pi}^{k}\right\rangle\right) \mathrm{d} \mu(\pi), \quad(g \in G)
$$

pour tout $A \in \mathcal{U}(\mathrm{g})$. A cette étape on espère bien que les vecteurs généralisés $a_{\pi}^{k}$ de Penney sont des vecteurs propres pour tous les opérateurs $\pi(\bar{A})$ avec $A \in \mathcal{U}(\mathfrak{g}, \tau)$, ce qui a été démontré dans [16] dans le cas nilpotent, et que la valeur propre nous fournit l'élément de $\mathrm{C}\left[\Gamma_{\tau}\right]^{H}$ associé à la classe de $A$ dans $D_{\tau}(G / H)$.

Une autre question à propos de l'étude de la réciproque en quelques sortes de la dernière question et qui se résume en:

QUESTION 2.2 (cf. [3, 7, 11, 18, 19]). Supposons que $G$ est nilpotent. Est-ce que la commutativité de cette algèbre des opérateurs différentiels $G$-invariants implique la finitude des multiplicités de $\tau$ presque partout?

Nous allons démontrer dans ce travail de nouveaux résultats concernant la conjecture (2.1) et la question (2.2). La majorité des résultats dans cette section concernent bien la deuxième question qui à eté formulée par M. Duflo dans un cadre plus général, reposée par Corwin-Greenleaf dans le cadre des groupes de Lie nilpotents. 
Il est à noter que cette dernière question possède une réponse négative dans le cas des groupes de Lie résolubles exponentiels (voir [7]).

Notons que dans le même cadre de groupes, nous avons étudié récemment la situation où le groupe $G$ est complètement résoluble (résoluble exponentiel avec des racines réelles) et où le sous-groupe induisant est une composante de Levi. Nous avons étudié l'espace des vecteurs généralisés semi-invariants et prouvé la conjecture (2.1) dans ce cas. (voir [2])

Nous commençons par étudier dans le paragraphe 3 le cas où le groupe $G$ est résoluble exponentiel et le sous-groupe induisant est normal. Cette situation a été étudiée dans le cas des groupes nilpotents par Baklouti et Ludwig dans [3]. Notre étude consiste à étendre ces résultats aux groupes résolubles exponentiels. Nous démontrons la conjecture (2.1) et nous répondons positivement à la question (2.2) dans cette situation et qu'elles sont équivalentes au fait que les multiplicités de $\tau$ sont finies presque partout. Nous étudions ensuite quelques exemples.

Dans le paragraphe 4, nous regardons de prés la question (2.2). Supposons alors que $\mathfrak{g}$ est nilpotente et que $\mathfrak{h}$ est une sous-algèbre de $\mathfrak{g}$ qui n'est pas nécessairement un idéal de g. Soit

$$
\mathfrak{h}=\mathfrak{f}_{0} \subset \mathfrak{f}_{1} \subset \cdots \subset \mathfrak{f}_{p-1} \subset \mathfrak{f}_{p}=\mathfrak{g}, \quad \operatorname{dim}\left(\mathfrak{f}_{r} / \mathfrak{f}_{r-1}\right)=1,
$$

une suite de Malcev relative à $\mathfrak{h}$. Soit $\mathfrak{g}^{\prime}=\mathfrak{f}_{p-1}$.

Il est bien clair que la résolution de cette question est fortement liée à la résolution de l'assertion suivante:

ASSERTION 2.3. Supposons que g est nilpotente et que $\mathfrak{h}$ est une sous-algèbre de $\mathfrak{g}$. Si les $H$-orbites dans $\Gamma_{\tau}$ sont génériquement non-saturées par rapport à $\mathfrak{g}^{\prime}$, alors il existe $W \in \mathcal{U}(\mathfrak{g}, \tau)$ s'écrivant comme

$$
W=X_{p} U+V
$$

avec $U, V \in \mathcal{U}\left(\mathrm{g}^{\prime}\right)$ vérifiant $U \notin \mathcal{U}(\mathrm{g}) \mathfrak{a}_{\tau}$.

Nous démontrons l'assertion (2.3) en ajoutant une hypothèse supplémentaire (Théorème (4.4) plus tard). Ensuite nous déterminons quelques hypothèses suffisantes pour résoudre la question (2.2). En particulier, nous donnons une réponse positive à cette question lorsque $\operatorname{dim} \mathfrak{h} \leqslant 2$ ou que $\operatorname{dim} \mathfrak{h}=\operatorname{dim} \mathfrak{b}(f)-1$ où $\mathfrak{b}(f)$ est une polarisation en $f$.

Nous croyons que le principe suivant doit être vrai. Ce principe dit:

Supposons que G est nilpotent et que $\mathfrak{h}$ est une sous-algèbre de $\mathfrak{g}$. Si $\mathfrak{h}$ est suffisamment petite, $\tau$ s'apprête à être à multiplicités infinies et l'algèbre $D_{\tau}(G / H)$ devient assez grande pour qu'elle soit non-commutative. Inversement, si h est suffisamment grande, l'algèbre $D_{\tau}(G / H)$ a tendance à la commutativité. Ceci étant, mais n'empêche qu'il existe des cas où $D_{\tau}(G / H)$ est commutative tandis que $\operatorname{dim} \mathfrak{h}=1$ et que $G$ n'est pas abélien, ou encore $D_{\tau}(G / H)$ est non-commutative tandis que $\operatorname{dim} \mathfrak{h}=\operatorname{dim} \mathfrak{b}(f)-1$, où $\mathfrak{b}(f)$ est une polarisation en $f$. 
Soit $K_{j}=\exp \left(\mathfrak{f}_{j}\right)$ et $\tau_{j}=\operatorname{Ind}_{H}^{K_{j}} \chi_{f}$ pour $j=1, \ldots, p$. Soit $D_{\tau_{j}}\left(K_{j} / H\right)$ l'algèbre des opérateurs différentiels définie pour les données $\mathfrak{f}_{j}, \mathfrak{h}$ et $f$. De la suite de Malcev (3), nous avons la série des inclusions suivantes:

$$
\mathrm{C} \cdot 1=D_{\tau_{0}}\left(K_{0} / H\right) \subset D_{\tau_{1}}\left(K_{1} / H\right) \subset \cdots \subset D_{\tau_{p}}\left(K_{p} / H\right)=D_{\tau}(G / H) .
$$

Ce qu'on attend à établir, c'est la propriété suivante:

ASSERTION 2.4. Supposons que g est nilpotente et que $\mathfrak{h}$ est une sous-algèbre de $\mathfrak{g}$. Soit

$$
\mathfrak{h}=\mathfrak{f}_{0} \subset \mathfrak{f}_{1} \subset \cdots \subset \mathfrak{f}_{p-1} \subset \mathfrak{f}_{p}=\mathfrak{g}, \quad \operatorname{dim}\left(\mathfrak{f}_{r} / \mathfrak{f}_{r-1}\right)=1
$$

la suite de Malcev relative à hy donnée dans (3). Au passage de $\mathfrak{f}_{j-1} a \grave{a} \mathfrak{f}_{j}$, l'algèbre $D_{\tau_{j-1}}\left(K_{j-1} / H\right)$ ne s'agrandirait pas si les $H$-orbites génériques dans

$$
\Gamma_{j}=\left\{l \in \mathfrak{f}_{j}^{\star}: l(Y)=f(Y), Y \in \mathfrak{h}\right\}
$$

sont saturées par rapport à $\mathfrak{f}_{j-1}$. Par contre, si elles sont non-saturées, l'algèbre en question s'agrandirait d'un générateur (dans un certain sens), $W \in \mathcal{U}(\mathfrak{g}, \tau) \cap \mathcal{U}\left(\mathfrak{f}_{j}\right)$, ayant la forme

$$
W=X_{j} U+V
$$

avec $U, V \in \mathcal{U}\left(\mathfrak{f}_{j-1}\right)$ vérifiant $U \notin \mathcal{U}(\mathfrak{g}) \mathfrak{a}_{\tau}$.

C'est le résultat fondamental dû à Corwin-Greenleaf dans le cas où $\tau$ serait à multiplicités finies. Nous confirmons alors cette assertion lorsque $\mathfrak{h}$ est une sousalgèbre de $\mathfrak{g}$ telle que $\operatorname{dim} \mathfrak{h} \leqslant 2$. Quelques exemples sont regardés le long de ce paragraphe.

Soit $\pi$ une représentation unitaire et irréductible d'un groupe de Lie résoluble exponentiel $G=\exp \mathfrak{g}$ associée à une orbite coadjointe $\Omega(\pi) \subset \mathfrak{g}^{\star}$ d'une forme linéaire $f$. Soit $H$ un sous-groupe analytique de $G$ d'algèbre de Lie $\mathfrak{h}$. Nous allons définir maintenant une certaine algèbre d'opérateurs différentiels associée à la représentation $\pi$. Pour deux sous-espaces $\mathcal{M}$ et $\mathcal{N}$ de l'algèbre enveloppante $\mathcal{U}(\mathfrak{g})$, on note

$$
c(\mathcal{M}, \mathcal{N})=\{A \in \mathcal{U}(\mathrm{g}):[A, \mathcal{M}] \subset \mathcal{N}\}
$$

le centralisateur de $\mathcal{M}$ modulo $\mathcal{N}$. Alors il est bien évident que $\operatorname{ker}(\mathrm{d} \pi)$ est un idéal bilatère de $c(\mathfrak{h}, \operatorname{ker}(\mathrm{d} \pi))=\mathcal{U}_{\pi}(\mathfrak{g})^{\mathfrak{h}}$. Soit $D_{\pi}(G)^{H}$ l'algèbre image par l'homomorphisme $\mathrm{d} \pi \mathrm{du}$ quotient $c(\mathfrak{h}, \operatorname{ker}(\mathrm{d} \pi)) / \operatorname{ker}(\mathrm{d} \pi)$. Ainsi,

$$
D_{\pi}(G)^{H} \simeq \mathcal{U}_{\pi}(\mathfrak{g})^{\mathfrak{h}} / \operatorname{ker}(\mathrm{d} \pi)=(\mathcal{U}(\mathfrak{g}) / \operatorname{ker}(\mathrm{d} \pi))^{H},
$$

où la dernière algèbre est l'algèbre des éléments $H$-invariants de $\mathcal{U}(\mathfrak{g}) / \operatorname{ker}(\mathrm{d} \pi)$.

L'objet du paragraphe 5 est l'étude de cette algèbre. Nous voulons comprendre la structure de l'algèbre $D_{\pi}(G)^{H}$, éventuellement la relation avec le spectre et les multiplicités de $\pi_{\mid H}$. En particulier, nous voulons répondre à la question suivante: Quand est-ce que $D_{\pi}(G)^{H}$ est commutative? C'est l'objet du paragraphe 5. 
Quand $G$ est nilpotent, nous donnons une description simple de cette algèbre en termes de l'algèbre enveloppante. Ensuite, nous étudions la situation où le sous-groupe $H$ est normal. Notre résultat principal dans cette section est le théorème suivant:

THÉORÈME 2.5. Supposons que $G$ est nilpotent et $H$ un sous-groupe normal de $G$. Alors, la finitude des multiplicités de $\pi_{\mid H}$ presque partout implique la commutativité de $D_{\pi}(G)^{H}$.

\section{Sur l'algèbre $D_{\tau}(G / H)$}

Le premier résultat concerne la situation où $H$ est un sous-groupe normal de $G$ :

THÉORÈME 3.1. Supposons que $G$ est un groupe de Lie résoluble exponentiel et $H$ est un sous-groupe normal de $G$, alors avec les mêmes notations, les trois assertions suivantes sont équivalentes:

(i) $m(\pi)=1, \mu$-presque partout.

(ii) L'algèbre $D_{\tau}(G / H)$ est abélienne.

(iii) $D_{\tau}(G / H) \simeq \mathrm{C}\left[\Gamma_{\tau}\right]^{H}$.

Ce théorème a été démontré dans [3] pour les groupes de Lie nilpotents. La généralisation de ce résultat aux cas des groupes résolubles exponentiels est l'objet de ce paragraphe. Il est bien connu $[20,27]$ que notre représentation monomiale $\tau=\operatorname{Ind}_{H}^{G} \chi$ est à multiplicités uniformément égales ou bien à un ou bien à l'infini. Soit $\mathfrak{a}$ un idéal non central minimal de $\mathfrak{g}$ contenu dans $\mathfrak{h}$. En calculant l'action de $\mathfrak{a}$ et en utilisant la semi-invariance imposée, on a étudié [17] (voir surtout la page 162) l'espace $\left(\mathcal{H}_{\pi}^{-\infty}\right)^{H, \chi}$ et ses éléments. Nous allons employer les mêmes arguments pour étudier l'algèbre $D_{\tau}(G / H)$.

Supposons maintenant que $m(\pi)<\infty$, ce qui veut dire que $m(\pi)=1 \mu$-presque partout comme $H$ est un sous-groupe normal de $G$. De ce qu'on a vu dans la section précédente, l'intersection $\Gamma_{\tau} \cap \Omega(\pi)$ est une seule $H$-orbite pour $\mu$-presque toute $\pi \in \hat{G}$. On en déduit que

$$
\mathfrak{b}=\mathfrak{h}^{f}=\{X \in \mathfrak{g}: f([X, \mathfrak{h}])=0\}
$$

est une polarisation commune pour $l \in \Gamma_{\tau}$ générique, et qui vérifie la condition de Pukanszky (cf. [17], page 162]). Il doit être noté que $\mathfrak{h} \subset \mathfrak{b}$. Réalisons $\pi \in \hat{G}$ générique associée à un élément $l$ de $\mathrm{g}^{\star} \operatorname{comme} \pi=\operatorname{Ind}_{B}^{G} \chi_{l}$ avec $B=\exp (\mathfrak{b})$.

Le lemme suivant est prouvé dans [3] dans le cas des groupes nilpotents, la preuve est faite par un raisonnement de récurrence. Nous allons démontrer maintenant le même résultat en suivant le même chemin que dans le cas nilpotent. Nous donnons aux lecteurs quelques idées utiles sur la preuve.

LEMME 3.2. On suppose que G est un groupe de Lie résoluble exponentiel et que $H$ est un sous-groupe normal de G. Alors, $\mathcal{U}(\mathfrak{g}, \tau)=\mathcal{U}(\mathfrak{b})+\mathcal{U}(\mathfrak{g}) \mathfrak{a}_{\tau}$. 
Preuve. Il est clair que $\mathfrak{b} \subset \mathcal{U}(\mathfrak{g}, \tau)$ et donc $\mathcal{U}(\mathfrak{b})$ l'est aussi, ainsi $\mathcal{U}(\mathfrak{g}, \tau) \supset$ $\mathcal{U}(\mathfrak{b})+\mathcal{U}(\mathfrak{g}) \mathfrak{a}_{\tau}$. Pour démontrer l'autre inclusion, nous employons une récurrence sur la dimension de g. Soit $z$ le centre de g. $\operatorname{Si} \operatorname{dim}(\mathfrak{h} \cap \mathfrak{z}) \leqslant 2$, nous avons $\mathfrak{p}=\mathfrak{h} \cap \mathfrak{z} \cap \operatorname{ker} f \neq\{0\}$, ce qui nous permet de descendre au quotient $\tilde{\mathfrak{g}}=\mathfrak{g} / \mathfrak{p}$.

Supposons que $\operatorname{dim}(\mathfrak{h} \cap z) \leqslant 1$, et de la même raison que $f_{\mid \mathfrak{g} \cap z} \neq 0$ si $\operatorname{dim}(\mathfrak{h} \cap \mathfrak{z})=1$. S'il existe un idéal minimal $\mathfrak{a}$ de $\mathfrak{g}$ inclus dans $\mathfrak{h}$ qui n'est pas central, alors $\operatorname{dim} a$ est un ou deux et l'on peut supposer que $f_{\left.\right|_{\mathfrak{a}}} \neq 0$ comme plus haut. Nous notons $\mathfrak{g}_{0}$ le centralisateur de $\mathfrak{a}$ dans $\mathfrak{g}$. Nous écrivons $\mathfrak{g}=\mathfrak{g}_{0} \oplus \mathbf{R} X$ avec un certain élément $X$ dont le choix sera précisé dans la suite. Nous écrivons $W \in \mathcal{U}(\mathfrak{g}, \tau)$ sous la forme

$$
W=\sum_{k=0}^{m} X^{k} V_{k}
$$

avec certains $V_{k} \in \mathcal{U}\left(\mathrm{g}_{0}\right)$.

(i) Soit $\operatorname{dim} \mathfrak{a}=1$. Choisissons $X$ et $Y$ de telle faç on qu'on ait $\mathfrak{a}=\mathbf{R} Y, f(Y)=1$ et $[X, Y]=Y$. Calculons $[W, Y]$ qui appartient à $\mathcal{U}(\mathfrak{g}) \mathfrak{a}_{\tau}$. Tout d'abord par un simple calcul, nous pouvons constater que pour tout entier $m$,

$$
\left[X^{m}, Y\right]=m X^{m-1} Y+Q
$$

où $Q \in \mathcal{U}(\mathfrak{g})$ de degré plus petit ou égale à $m-2$ en $X$. Alors il vient que

$$
[W, Y]=m X^{m-1} V_{m} Y+A \equiv-\operatorname{im} X^{m-1} V_{m}+A\left(\operatorname{modulo} \mathcal{U}(\mathfrak{g}) \mathfrak{a}_{\tau}\right)
$$

avec un certain $A \in \mathcal{U}(\mathfrak{g})$ de degré plus petit ou égale à $m-2$ par rapport à $X$. Il en résulte que $V_{m} \in \mathcal{U}(\mathfrak{g}) \mathfrak{a}_{\tau}$ et inductivement que $W \in \mathcal{U}\left(\mathfrak{g}_{0}\right)$ modulo $\mathcal{U}(\mathfrak{g}) \mathfrak{a}_{\tau}$.

(ii) Soit $\operatorname{dim} \mathfrak{a}=2$. Choisissons $X$ et $Y_{1}, Y_{2}$ de telle façon qu'on ait $\mathfrak{a}=\mathbf{R} Y_{1} \oplus \mathbf{R} Y_{2}$ et

$$
\left[X, Y_{1}\right]=Y_{1}-\alpha Y_{2},\left[X, Y_{2}\right]=Y_{2}+\alpha Y_{1}
$$

avec un certain $\alpha$ réel non nul. Calculons $\left[W, Y_{1}\right]$ et $\left[W, Y_{2}\right]$ qui appartiennent à $\mathcal{U}(\mathfrak{g}) \mathfrak{a}_{\tau}$. Suivant le même chemin que dans (i), nous pouvons constater que

$$
\begin{aligned}
& {\left[W, Y_{1}\right]=m X^{m-1} V_{m}\left(Y_{1}-\alpha Y_{2}\right)+A_{1}} \\
& {\left[W, Y_{2}\right]=m X^{m-1} V_{m}\left(Y_{2}+\alpha Y_{1}\right)+A_{2}}
\end{aligned}
$$

avec certains $A_{1}, A_{2} \in \mathcal{U}(\mathfrak{g})$ de degré plus petit ou égale à $m-2$ par rapport à $X$. Quitte à supposer que $f_{\mid \mathfrak{a}} \neq 0$, on en conclut que $V_{m} \in \mathcal{U}(\mathfrak{g}) \mathfrak{a}_{\tau}$ et successivement que $W \in \mathcal{U}\left(\mathrm{g}_{0}\right)$ modulo $\mathcal{U}(\mathrm{g}) \mathfrak{a}_{\tau}$.

Pour terminer avec ces deux premiers cas, nous notons que $\mathfrak{h} \subset \mathfrak{b} \subset \mathfrak{g}_{0}$, donc en appliquant l'hypothèse de récurrence, nous avons que

$$
W \in \mathcal{U}(\mathfrak{b})+\mathcal{U}\left(\mathfrak{g}_{0}\right) \mathfrak{a}_{\tau}+\mathcal{U}(\mathfrak{g}) \mathfrak{a}_{\tau} \subset \mathcal{U}(\mathfrak{b})+\mathcal{U}(\mathfrak{g}) \mathfrak{a}_{\tau} .
$$

S'il n'existe aucun idéal minimal contenu dans $\mathfrak{h}$ qui soit non central, alors $\operatorname{dim}(\mathfrak{h} \cap \mathfrak{z})=1$ sous notre hypothèse. Soit $\mathfrak{h} \cap \mathfrak{z}=\mathbf{R} Z$ avec $f(Z)=1$. En prenant 
dans $\mathfrak{h}$ un idéal non central minimal $\mathfrak{a}$ de $\mathfrak{g}$, on se trouve dans l'une des situations suivantes. Nous noterons $\mathfrak{g}_{0}$ le centralisateur de $\mathfrak{a}$ dans $\mathfrak{g}$.

Supposons d'abord que $\operatorname{dim} \mathfrak{a}=2$. Soit $\operatorname{dim} \mathfrak{g} / \mathfrak{g}_{0}=2$. Il existe des éléments $Y$ et $X_{1}, X_{2}$ de telle façon qu'on ait $\mathfrak{a}=\mathbf{R} Y \oplus \mathbf{R} Z, f(Y)=0$ et $\left[X_{1}, Y\right]=Z,\left[X_{2}, Y\right]=$ $Y$. Nous prenons $W \in \mathcal{U}(\mathfrak{g}, \tau)$. $W$ s'écrit comme

$$
W=\sum_{k=0}^{m} X_{1}^{k} V_{k}
$$

où $V_{k}=\sum_{j=0}^{m_{k}} X_{2}^{j} U_{k, j}$ avec $U_{k, j} \in \mathcal{U}\left(\mathrm{g}_{0}\right)$. Calculons l'élément $[W, Y]$ qui appartient à $\mathcal{U}(\mathrm{g}) \mathfrak{a}_{\tau}$. Nous remarquons d'abord que $\left[V_{k}, Y\right] \in \mathcal{U}(\mathrm{g}) \mathfrak{a}_{\tau}$. En effet,

$$
\left[V_{k}, Y\right]=m_{k} X_{2}^{m_{k}-1} U_{k, m_{k}} Y+A Y
$$

avec un certain $A \in \mathcal{U}(\mathrm{g})$ de degré plus petit ou égale à $m_{k}-2$ en $X_{2}$. Nous avons donc modulo $\mathcal{U}(\mathfrak{g}) \mathfrak{a}_{\tau}$,

$$
[W, Y] \equiv m X_{1}^{m-1} V_{m} Z+B \equiv-\mathrm{im} X_{1}^{m-1} V_{m}+B
$$

avec un certain $B \in \mathcal{U}(\mathrm{g})$ de degré plus petit ou égale à $m-2$ en $X_{1}$. D'où $V_{m} \in \mathcal{U}(\mathfrak{g}) \mathfrak{a}_{\tau}$, et en continuant de suite, on conclut que $W$ est représenté par un élément de $\mathcal{U}\left(\mathfrak{g}_{1}\right)$ avec $\mathfrak{g}_{1}=\mathbf{R} X_{2} \oplus \mathfrak{g}_{0}=\mathfrak{a}^{f}$ qui est une sous-algèbre de $\mathfrak{g}$. Nous appliquons donc l'hypothèse de récurrence sur $\mathfrak{g}_{1}$ comme plus haut pour obtenir le résultat. $\mathrm{Si} \operatorname{dim} \mathfrak{g} / \mathfrak{g}_{0}=1, X_{2}$ n'apparaîtrait pas et le procédé ci-dessus deviendrait quasiment direct.

Supposons enfin que $\operatorname{dim} \mathfrak{a}=3$. Il existe des éléments $X_{0}, X_{1}, X_{2}, Y_{1}, Y_{2}$ tels que $\mathfrak{a}=\mathbf{R} Y_{1} \oplus \mathbf{R} Y_{2} \oplus \mathbf{R} Z, f\left(Y_{1}\right)=f\left(Y_{2}\right)=0$ et $\left[X_{i}, Y_{j}\right]=\delta_{i, j} Z,(1 \leqslant i, j \leqslant 2)$,

$$
\left[X_{0}, Y_{1}\right]=Y_{1}-\alpha Y_{2}, \quad\left[X_{0}, Y_{2}\right]=Y_{2}+\alpha Y_{1}(0 \neq \alpha \in \mathbf{R}) .
$$

Etant donné $W \in \mathcal{U}(\mathfrak{g}, \tau)$, nous allons faire le même calcul. Nous écrivons

$$
W=\sum_{k=0}^{m} X_{1}^{k} V_{k}
$$

où $V_{k}=\sum_{j=0}^{m_{k}} X_{2}^{j} U_{k, j}$ avec $U_{k, j} \in \mathcal{U}\left(\tilde{\mathfrak{g}}_{0}\right)$, où $\tilde{\mathfrak{g}}_{0}$ est la sous-algèbre de codimension deux dans $\mathfrak{g}$ donnée par $\tilde{\mathfrak{g}}_{0}=\mathbf{R} X_{0} \oplus \mathfrak{g}_{0}=\mathfrak{a}^{f}$. Tout d'abord nous suivons exactement le même chemin pour voir que $\left[U_{k, j}, Y_{1}\right],\left[U_{k, j}, Y_{2}\right]$ appartiennent à $\mathcal{U}(\mathrm{g}) \mathfrak{a}_{\tau}$. Puis

$$
\left[V_{k}, Y_{2}\right] \equiv m_{k} X_{2}^{m_{k}-1} U_{k, m_{k}} Z+A_{2} \equiv-\operatorname{im}_{k} X_{2}^{m_{k}-1} U_{k, m_{k}}+A_{2}\left(\operatorname{modulo} \mathcal{U}(\mathrm{g}) \mathfrak{a}_{\tau}\right),
$$

où $A_{2}$ dénote un élément de $\mathcal{U}(\mathfrak{g})$ de degré plus petit ou égale à $m_{k}-2$ par rapport à $X_{2}$. Cela entraîne que nous pouvons supposer que $V_{k} \in \mathcal{U}\left(\tilde{\mathfrak{g}}_{0}\right)$ car

$$
\left[W, Y_{2}\right] \equiv \sum_{k=0}^{m} X_{1}^{k}\left[V_{k}, Y_{2}\right] \in \mathcal{U}(\mathfrak{g}) \mathfrak{a}_{\tau} .
$$

Ceci étant, calculons $\left[W, Y_{1}\right]$ qui appartient à $\mathcal{U}(\mathfrak{g}) \mathfrak{a}_{\tau}$ :

$$
\left[W, Y_{1}\right] \equiv m X_{1}^{m-1} V_{m} Z+A_{1} \equiv-\mathrm{im} X_{1}^{m-1} V_{m}+A_{1}\left(\operatorname{modulo} \mathcal{U}(\mathfrak{g}) \mathfrak{a}_{\tau}\right),
$$


ici $A_{1}$ dénote un élément de $\mathcal{U}(\mathrm{g})$ de degré plus petit ou égale à $m-2$ en $X_{1}$. Il en résulte finalement que $V_{m} \in \mathcal{U}(\mathfrak{g}) \mathfrak{a}_{\tau}$ et l'on se ramène au cas où $m=0$.

Compte tenu de ce qui précède, il nous suffit d'appliquer la récurrence sur $\tilde{\mathfrak{g}}_{0}$ comme plus haut pour obtenir le résultat cherché.

Rappellons un résultat de [17]. Si $m(\pi)<\infty \mu$-presque partout, on construit pour $l \in \Gamma_{\tau}$ générique la représentation irréductible $\pi=\Theta(l)=\operatorname{Ind}_{B}^{G} \chi_{l}$ au moyen de $B=\exp (\mathfrak{b}), \mathfrak{b}=\mathfrak{h}^{f}$. Alors ([17], page 162), $\left(\mathcal{H}_{\pi}^{-\infty}\right)^{H, \chi}=\mathbb{C} \delta_{\pi}$, où $\delta_{\pi}$ désigne la mesure de dirac pour $\pi$. Par ailleurs $\left(\mathcal{H}_{\rho}^{-\infty}\right)^{H, \chi}=\{0\}$ pour $\rho \in \hat{G}$ vérifiant $\Gamma_{\tau} \cap \Omega(\rho)=\emptyset$.

Définissons l'application $\quad \kappa: \mathfrak{g} \rightarrow \mathcal{U}(\mathfrak{g}) \quad$ par $\quad \kappa(X)=X+\frac{1}{2} \operatorname{Tr} \operatorname{ad}_{\mathfrak{g} / \mathfrak{b}} X, X \in \mathfrak{g}$. Puisque $\kappa\left(\left[X_{1}, X_{2}\right]\right)=\kappa\left(X_{1}\right) \kappa\left(X_{2}\right)-\kappa\left(X_{2}\right) \kappa\left(X_{1}\right)$ pour tous $X_{1}, X_{2} \in \mathfrak{g}, \kappa$ s'étend en un homomorphisme de $\mathcal{U}(\mathrm{g})$ dans $\mathcal{U}(\mathrm{g})$, noté encore $\kappa$.

LEMME 3.3. On suppose que G est un groupe de Lie résoluble exponentiel et que $H$ est un sous-groupe normal de G. Alors

(i) Pour tout $X \in \mathfrak{b}$, nous avons $\pi(X) \delta_{\pi}=\kappa(X)($ il $) \delta_{\pi}$.

(ii) Pour tout $W \in \mathcal{U}(\mathfrak{b})$, nous avons $\pi(W) \delta_{\pi}=\beta^{-1}(\kappa(W))($ il $) \delta_{\pi}$.

Preuve. Soit $X \in \mathfrak{b}$. Pour $\varphi \in \mathcal{H}_{\pi}^{\infty}$, nous avons

$$
\begin{aligned}
& \left\langle\pi(X) \delta_{\pi},\right\rangle=\left\langle\delta_{\pi}, \pi(-X) \varphi\right\rangle \\
& =\overline{(\pi(-X) \varphi)(e)}=\overline{\frac{\mathrm{d}}{\mathrm{d} t} \varphi(\exp (t X))_{\mid t=0}} \\
& =\overline{\left(\frac{\mathrm{d}}{\mathrm{d} t} e^{-i t l(X)+\frac{t}{2} \operatorname{Tr} \operatorname{ad}_{\mathbb{g} / b} X}\right)_{\mid t=0} \varphi(e)} \\
& =\left(\mathrm{il}(X)+\frac{1}{2} \operatorname{Tr} \operatorname{ad}_{\mathfrak{g} / \mathfrak{b}} X\right) \overline{\varphi(e)} \\
& =\left\langle\kappa(X)(i l) \delta_{\pi}, \varphi\right\rangle \text {, }
\end{aligned}
$$

d'où découle (i). L'assertion (ii) s'ensuit de (i) puisque $\left[X_{1}, X_{2}\right](i l)=0$ quelques soient $X_{1}, X_{2}$ dans $\mathfrak{b}$.

Le Lemme (3.3) nous fournit l'homomorphisme d'algèbres:

$$
D_{\tau}(G / H) \simeq \mathcal{U}(\mathfrak{b}) / \mathcal{U}(\mathfrak{b}) \mathfrak{a}_{\tau} \ni[W] \mapsto P_{W}(l)=\beta^{-1}(\kappa(W))(i l) \in \mathbb{C}\left[\Gamma_{\tau}\right]^{H},
$$

où $[W]$ dénote l'image canonique de $W$. En plus, d'après la formule (2), cet homomorphisme s'avère injectif. D'autre part, $\Gamma_{\tau} \cap \Omega(\pi)=H \cdot l=B \cdot l=l+\mathfrak{b}^{\perp}$, ce qui entraîne que $\mathrm{C}\left[\Gamma_{\tau}\right]^{H}=S(\mathfrak{b}) / S(\mathfrak{b}) \overline{\mathfrak{a}_{\tau}}$ et donc que l'homomorphisme défini ci-desus est surjectif. Par conséquent nous sommes arrivés à la conclusion suivante:

Si $\tau$ est à multiplicités finies, alors l'algèbre $D_{\tau}(G / H)$ est isomorphe à $\mathrm{C}\left[\Gamma_{\tau}\right]^{H}$. En particulier, $D_{\tau}(G / H)$ est commutative. 
Nous allons rappeler maintenant un résultat bien connu, et qui sera utilisé plus tard à maintes reprises. Supposons que $\mathfrak{g}$ est résoluble exponenentielle. Soient $\mathfrak{g}_{0}$ un idéal de $\mathfrak{g}$ tel que $\operatorname{dim} \mathfrak{g} / \mathfrak{g}_{0}=1$ et $G_{0}=\exp \mathfrak{g}_{0}$. Soit $p: \mathfrak{g}^{\star} \longrightarrow \mathfrak{g}_{0}^{\star}$ la projection canonique. Par rapport à $\mathfrak{g}_{0}$, les orbites coadjointes de $G$, donc aussi les représentations unitaires et irréductibles de $G$, se classent en deux catégories. On note $G(f)$ le stabilisateur de $f \in \mathfrak{g}^{\star}$ dans $G$, dont l'algèbre de Lie s'écrit $\mathfrak{g}(f)$. Dans la suite, on dit que l'orbite $\Omega$ de $f$ est une orbite saturée par rapport à $\mathrm{g}_{0}$ (respectivement non saturée par rapport à $\left.\mathfrak{g}_{0}\right)$ si $\mathfrak{g}(f) \subset \mathfrak{g}_{0}$ (respectivement si $\left.\mathfrak{g}(f) \not \subset \mathfrak{g}_{0}\right)$. Cette notion de saturation est équivalente à celle définie dans le premier paragraphe lorsque le groupe $G$ est nilpotent.

THÉORÈME 3.4 (cf. [20, 27]). Supposons que $G$ est résoluble exponentiel et $G_{0}$ un sous-groupe normal de codimension un dans $G$. Soient $\Omega$ une orbite coadjointe de $G, f \in \Omega$ et $\pi(\Omega)$ la représentation unitaire et irréductible de $G$, associée à $\Omega$. On a l'alternative suivante:

(i) Si $\Omega$ est une orbite saturée par rapport à $\mathrm{g}_{0}$, alors $p(\Omega)$ est réunion de $G_{0}$-orbites $\omega_{t}$ à un paramètre $t \in \mathbf{R}, \omega_{t}=\exp (t X) \cdot \omega_{0}$ avec $X \notin \mathrm{g}_{0}$ et $\Omega=p^{-1}(p(\Omega))$. D'autre part, $\pi(\Omega)_{\left.\right|_{0}} \simeq \int_{\mathbf{R}}^{\oplus} \pi\left(\omega_{t}\right) \mathrm{d} t$ tandis que $\operatorname{Ind}_{G_{0}}^{G} \pi\left(\omega_{t}\right)$ est irréductible.

(ii) Si $\Omega$ est une orbite non saturée par rapport à $\mathrm{g}_{0}$, alors $p(\Omega)$ est une $G_{0}$-orbite, notée $\omega$, et $p^{-1}(\omega)$ est réunion de G-orbites $\Omega_{t}$ à un paramètre $t \in \mathbf{R}$. D'autre part, $\pi\left(\Omega_{t}\right)_{\left.\right|_{0}}$ est irréductible et $\operatorname{Ind}_{G_{0}}^{G} \pi(\omega) \simeq \int_{\mathbf{R}}^{\oplus} \pi\left(\Omega_{t}\right) \mathrm{d} t$.

Pour terminer la preuve, nous allons prouver le lemme suivant:

LEMME 3.5. On suppose que $G$ est un groupe de Lie résoluble exponentiel et que $H$ est un sous-groupe normal de G. Si $\tau$ est à multiplicités infinies, alors l'algèbre $D_{\tau}(G / H)$ n'est pas commutative.

Preuve. Soit $\mathfrak{g}=\left(\mathfrak{a}_{j}\right)_{j=0}^{n}$ une bonne suite de sous-algèbres de $\mathfrak{g}$, c'est à dire une suite de sous-algèbres telle que:

$$
\{0\}=\mathfrak{a}_{0} \subset \mathfrak{a}_{1} \subset \cdots \subset \mathfrak{a}_{n-1} \subset \mathfrak{a}_{n}=\mathfrak{g},
$$

pour tout $j, 1 \leqslant j \leqslant n, \operatorname{dim} \mathfrak{a}_{j} / \mathfrak{a}_{j-1}=1$ et telle que, si $\mathfrak{a}_{j}$ n'est pas un idéal de $\mathfrak{g}, \mathfrak{a}_{j-1}$ et $\mathfrak{a}_{j+1}$ sont toutes les deux des idéaux de $\mathfrak{g}$ et $\mathfrak{a}_{j+1} / \mathfrak{a}_{j-1}$ est un $\mathfrak{g}$-module irréductible. Dans ce cas $\mathfrak{a}_{j-1}$ est toujours un idéal de $\mathfrak{a}_{j}$ pour $1 \leqslant j \leqslant n$. Fixons $\mathfrak{g}=\left(\mathfrak{a}_{j}\right)_{j=0}^{n}$ une bonne suite de sous-algèbres de $\mathfrak{g}$, comme $\mathfrak{h}$ est un idéal de $\mathfrak{g}$, on peut choisir $\mathfrak{g}$ de faç on que $\mathfrak{h}=\mathfrak{a}_{r}$ pour certain $r \in\{1, \ldots, n\}$. Dans ce cas, nous avons que $\mathfrak{f}_{0}=\mathfrak{h}$ et $\mathfrak{f}_{j}=\mathfrak{a}_{j+r}$ pour $j=0, \ldots, p$ avec $p+r=n$.

Nous rappelons maintenant le résultat suivant: Soit

$$
\begin{array}{r}
I^{H}=\left\{j \in\{1, \ldots, p\} ; \text { les } K_{j} \text {-orbites génériques dans } \Gamma_{\tau \mid f_{j}}\right. \\
\text { sont saturées par rapport à } \left.\mathfrak{f}_{j-1}\right\},
\end{array}
$$

et

$$
\begin{gathered}
I_{0}^{H}=\left\{j \in I^{H} ; \text { les } H \text {-orbites génériques dans } \Gamma_{\tau \mid f_{j}}\right. \text { sont saturées } \\
\text { par rapport à } \left.\mathfrak{f}_{j-1}\right\} .
\end{gathered}
$$


Nous avons alors la caractérisation suivante concernant les multiplicités de $\tau$.

PROPOSITION 3.5.1. Supposons que G est nilpotent et $H$ est un sous-groupe de $G$ (ou que G est résoluble exponentiel et que $H$ est un sous-groupe normal de G). Alors, les conditions suivantes sont équivalentes:

(i) Les multiplicités de $\tau=\operatorname{Ind}_{H}^{G} \chi_{f}$ sont finies.

(ii) $I_{0}^{H}=I^{H}$.

La proposition a été démontrée dans [3] dans le cas où $G$ est nilpotent et $H$ est un sous-groupe de $G$. La preuve pourra être simplement adaptée au cas où $G$ est résoluble exponentiel et que $H$ est un sous-groupe normal de $G$.

Revenons maintenant à la preuve du lemme. Il suffit d'étudier le cas où il existe dans $\mathfrak{g}$ un idéal $\mathfrak{g}_{0}$ de codimension un contenant $\mathfrak{h}$ et tel que la représentation induite $\tau_{0}=\operatorname{Ind}_{H}^{G_{0}} \chi, G_{0}=\exp \left(g_{0}\right)$, soit à multiplicités finies. Pour $l \in \Gamma_{\tau}$ arbitraire, on voit que $\Gamma_{\tau} \cap G \cdot l=G^{\prime}\left(f_{\mid \mathfrak{h}}\right) \cdot l$, où $G\left(f_{\mid \mathfrak{h}}\right)$ dénote le stabilisateur de $f_{\mid \mathfrak{l}} \in \mathfrak{h}^{*}$ dans $G$ (cf. [20,27]). Tenons compte du fait que $G\left(f_{\mid \mathfrak{l}}\right)$ est un sous-groupe connexe de $G$, i.e $G\left(f_{\mid \mathfrak{G}}\right)=\exp \left(\mathfrak{g}\left(f_{\mid \mathfrak{G}}\right)\right)$ ayant l'algèbre de Lie $\mathfrak{g}\left(f_{\mid \mathfrak{G}}\right)$. Alors $\mu$-presque toutes les orbites $G \cdot l$ sont saturées dans la direction de $\mathfrak{g}_{0}^{\perp}$ (cf. [20]). D'aprés la proposition 3.5.1, les $H$-orbites génériques dans $\Gamma_{\tau}$ doivent être non-saturées par rapport à $\mathfrak{g}_{0}$, il existe alors $X \in \mathfrak{g} \backslash \mathfrak{g}_{0}$ appartenant à $\mathfrak{g}\left(f_{\mid \mathfrak{h}}\right)$. D'autre part, comme $\tau_{0}$ est à multiplicités finies, $\mathfrak{g}_{0}\left(f_{\mid \mathfrak{h}}\right)=\mathfrak{h}+\mathfrak{g}_{0}\left(l_{\mid \mathfrak{g}_{0}}\right)$ est une polarisation de $\mathfrak{g}_{0}$ en $l_{\mid \mathfrak{g}_{0}}$ et donc une polarisation de $\mathfrak{g}$ en $l$. Nous en déduisons que $\mathfrak{h}$ est inclus strictement dans $\mathfrak{g}_{0}\left(f_{\mid \mathfrak{h}}\right)$ comme $\mathfrak{g}_{0}\left(f_{\mid \mathfrak{h}}\right)$ st isotrope et maximale. Il existe donc $Y \in \mathfrak{g}_{0}\left(l_{\mathfrak{g}_{0}}\right)$ vérifiant $[X, Y] \notin \mathfrak{h} \cap \operatorname{ker} f$. Evidement $X, Y$ appartiennent à $\mathcal{U}(\mathfrak{g}, \tau)$ et la condition $[X, Y] \notin \mathfrak{h} \cap \operatorname{ker} f$ signifie qu'ils ne commutent pas entre eux.

COROLLAIRE 3.6. On suppose que G est un groupe de Lie résoluble exponentiel et que $H$ est un sous-groupe normal de $G$. Alors l'algèbre $D_{\tau}(G / H)$ est commutative si et seulement si $\tau$ est à multiplicités finies.

Cependant, nous aurons besoin dans la suite de la proposition suivante:

PROPOSITION 3.7. On suppose que G est un groupe de Lie résoluble exponentiel. Soit $\mathfrak{h}$, une polarisation réelle au point $f \in \mathfrak{g}^{\star}$ vérifiant la condition de Pukanszky. Alors l'algèbre $D_{\tau}(G / H)$ est triviale.

Preuve. Procédons comme d'habitude par récurrence sur la dimension de $\mathfrak{g}$ pour montrer que $\mathcal{U}(\mathfrak{g}, \tau)=\mathrm{C} 1+\mathcal{U}(\mathfrak{g}) \mathfrak{a}_{\tau}$. Pourvu que $f$ s'annule sur un idéal non trivial $\mathfrak{a}$ de $\mathfrak{g}$, nous pouvons descendre au quotient $\mathfrak{g} / \mathfrak{a}$ auquel s'applique l'hypothèse de récurrence. Supposons que $f$ ne s'annule sur aucun idéal non trivial de g. Comme dans la démonstration du Lemme 3.2, prenons un idéal non central minimal $\mathfrak{a}$ de $\mathfrak{g}$. Si $\mathfrak{a} \subset \mathfrak{h}$, la réduction faite dans la démonstration du Lemme 3.2 nous permet de nous ramener à la sous-algèbre $\mathfrak{a}^{f}$ à laquelle s'applique l'hypothèse de récurrence. 
Si $\mathfrak{a} \not \subset \mathfrak{h}$, nous avons d'après b) et c) de la page $124 \mathrm{du}[5]$ que $\operatorname{dim} \mathfrak{h} /\left(\mathfrak{h} \cap \mathfrak{a}^{f}\right)=$ $\operatorname{dim} \mathfrak{a} /(\mathfrak{h} \cap \mathfrak{a})$ et nous en déduisons que

$$
\mathcal{U}(\mathfrak{g}, \tau)=\left(\mathcal{U}(\mathfrak{g}, \tau) \cap \mathcal{U}\left(\mathfrak{a}^{f}\right)\right)+\mathcal{U}(\mathfrak{g}) \mathfrak{a}_{\tau} .
$$

En effet, il nous suffit d'examiner le cas où $\operatorname{dim} \mathfrak{a}=3$ et $\operatorname{dim}(\mathfrak{a} \cap \mathfrak{h})=2$. Donc la partie $z$ de $\mathfrak{a}$ qui est centrale dans $\mathfrak{g}$ est unidimensionnelle et il existe des éléments $X_{1}, X_{2}, Y_{1}, Y_{2}, Z$ tels que $\mathfrak{z}=\mathbf{R} Z, \mathfrak{a}=\mathbf{R} Y_{1} \oplus \mathbf{R} Y_{2} \oplus \mathfrak{\jmath}, \mathfrak{h}=\mathbf{R} X_{1}+\left(\mathfrak{h} \cap \mathfrak{a}^{f}\right), \mathfrak{h} \cap \mathfrak{a}=$ $\left.\mathbf{R} Y_{2} \oplus\right\}, f(Z)=1, f\left(Y_{i}\right)=f\left(X_{i}\right)=0$ et $\left[X_{i}, Y_{j}\right]=\delta_{i, j} Z,(1 \leqslant i, j \leqslant 2)$, en plus $\mathfrak{g}=$ $\mathbf{R} X_{1} \oplus \mathbf{R} X_{2} \oplus \mathfrak{a}^{f}$. Etant donné $W \in \mathcal{U}(\mathfrak{g}, \tau)$, écrivons

$$
W=\sum_{k=0}^{m} X_{2}^{k} V_{k}
$$

modulo $\mathcal{U}(\mathrm{g}) \mathfrak{a}_{\tau}$ avec certains $V_{k} \in \mathcal{U}\left(\mathfrak{a}^{f}\right)$. Compte tenu du fait que $\left[Y_{2}, W\right] \in \mathcal{U}(\mathfrak{g}) \mathfrak{a}_{\tau}$, nous voyons que $V_{m}$ appartient au centralisateur de $\mathfrak{a}$. S'il en est ainsi, nous avons que

$$
\left(\operatorname{ad}\left(Y_{2}\right)\right)^{m}(W) \equiv m ! i^{m} V_{m} \in \mathcal{U}(\mathfrak{g}) \mathfrak{a}_{\tau},
$$

ce qui nous donne l'assertion cherchée.

Pour tout $l \in \Gamma_{\tau}$, introduisons $\mathfrak{h}_{l}=\left(\mathfrak{h} \cap \mathfrak{a}^{l}\right)+\mathfrak{a}$, qui est une autre polarisation au point $l \in \mathfrak{g}^{*}$ vérifiant la condition de Pukanszky. Soient $H_{l}=\exp \left(\mathfrak{h}_{l}\right)$ et $\tau_{l}=\operatorname{ind}_{H_{l}}^{G} \chi_{l}$. Cela étant, il est évident que

$$
\mathcal{U}(\mathfrak{g}, \tau) \cap \mathcal{U}\left(\mathfrak{a}^{l}\right) \subset \mathcal{U}\left(\mathfrak{a}^{l}, \tau_{l}\right)=\mathrm{C} 1+\mathcal{U}\left(\mathfrak{a}^{l}\right) \mathfrak{a}_{\tau_{l}},
$$

car $\mathfrak{a} \subset \mathfrak{h}_{l}$. D'où il nous suffit de montrer que $\mathcal{U}\left(\mathfrak{a}^{f}\right) \mathfrak{a}_{\tau_{f}} \cap \mathcal{U}(\mathfrak{g}, \tau) \subset \mathcal{U}(\mathfrak{g}) \mathfrak{a}_{\tau}$. Soient $\mathfrak{g}_{0}=\{X \in \mathfrak{g}:[X, \mathfrak{a}] \subset \mathfrak{z}\}$ et $\mathfrak{f}$ le centralisateur de $\mathfrak{a}$ dans $\mathfrak{g}$. Donc si $\mathfrak{g} \neq \mathfrak{g}_{0}, \mathfrak{g}_{0}$ est un idéal de codimension 1 et $f$ est celui de codimension plus petite ou égale à 3 . Lorsque $\mathfrak{h} \not \subset \mathfrak{g}_{0}$, chaque élément de $\mathcal{U}\left(\mathfrak{a}^{f}\right) \mathfrak{a}_{\tau_{f}} \cap \mathcal{U}(\mathfrak{g}, \tau)$ admet un représentant modulo $\mathcal{U}(\mathfrak{g}) \mathfrak{a}_{\tau}$ dans $\mathcal{U}(\mathfrak{f}) \mathfrak{a}_{\tau^{\prime}} \cap \mathcal{U}(\mathfrak{g}, \tau)$, où, en utilisant une base $\left\{U_{j}\right\}_{j=1}^{p}$ de $(\mathfrak{h} \cap \mathfrak{f})+\mathfrak{a}$, nous avons posé

$$
\mathfrak{a}_{\tau^{\prime}}=\sum_{j=1}^{p} \mathrm{C}\left(U_{j}+i f\left(U_{j}\right)-\frac{1}{2} \operatorname{Trad}_{\mathfrak{f} /(\mathfrak{G} \cap \mathfrak{f})}\left(U_{j}\right)\right) .
$$

Lorsque $\mathfrak{h} \subset \mathfrak{g}_{0}$, en écrivant chaque élément $W \in \mathcal{U}\left(\mathfrak{a}^{f}\right) \mathfrak{a}_{\tau_{f}} \cap \mathcal{U}(\mathfrak{g}, \tau)$ sous une forme

$$
W=\sum_{k=0}^{m} X_{0}^{k} V_{k}
$$

avec certains $V_{k} \in \mathcal{U}(\mathfrak{f}) \mathfrak{a}_{\tau_{f}}$ et un certain $X_{0} \in \mathfrak{a}^{f} \backslash \mathfrak{f}$, nous voyons que $V_{m} \in \mathcal{U}(\mathfrak{f}) \mathfrak{a}_{\tau^{\prime}} \cap$ $\mathcal{U}(\mathfrak{g}, \tau)$ modulo $\mathcal{U}(\mathfrak{g}) \mathfrak{a}_{\tau}$.

Tout compte fait, il nous suffit de vérifier l'inclusion

$$
\mathcal{U}(\mathfrak{f}) \mathfrak{a}_{\tau^{\prime}} \cap \mathcal{U}(\mathfrak{g}, \tau) \subset \mathrm{C} 1+\mathcal{U}(\mathfrak{g}) \mathfrak{a}_{\tau} .
$$

Mais cette dernière s'ensuit du fait que $\mathcal{U}(\mathfrak{f}) \mathfrak{a}_{\tau^{\prime}} \cap \mathcal{U}(\mathfrak{g}, \tau) \subset \bigcap_{l \in \Gamma_{\tau}}\left(\mathbb{C} 1+\mathcal{U}\left(\mathfrak{a}^{l}\right) \mathfrak{a}_{\tau_{l}}\right)$. 
Soient $\mathfrak{h}$ une polarisation réelle au point $f \in \mathfrak{g}^{\star}$ vérifiant la condition de Pukanszky et $\tau=\operatorname{Ind}_{H}^{G} \chi_{f}$ la représentation unitaire et irréductible de $G$ associée à l'orbite coadjointe $\Omega(\tau)=G \cdot f$. Soient $W$ un élément central de $\mathcal{U}(\mathfrak{g})$ et $W \mapsto W^{\diamond}$ l'antiautomorphisme principal de $\mathcal{U}(\mathrm{g})$. Il existe un scalaire $\lambda(W)$ tel que

$$
W^{\diamond} \equiv \lambda(W)
$$

modulo $\mathcal{U}(\mathfrak{g}) \mathfrak{a}_{\tau}$. En plus ce scalaire $\lambda(W)$ ne dépend ni du choix de la polarisation ni de $f \in \Omega(\tau)$ elle-même, il ne dépend que de l'orbite $\Omega(\tau)$, et nous avons que $\mathrm{d} \tau(W) \varphi=\lambda(W) \varphi$ pour tout $\varphi \in \mathcal{H}_{\tau}^{+\infty}$.

EXEMPLE 3.8. Soit $\mathfrak{g}=\operatorname{vect}(A, X, Y, Z)_{\mathbf{R}}$ où les uniques crochets non-nuls sont

$$
[A, X]=-X,[A, Y]=Y,[X, Y]=Z .
$$

On constate aussitõt que $W=A Z-X Y$ est un élément central dans $\mathcal{U}(\mathrm{g})$. Soient $f=\alpha Z^{\star}+\beta A^{\star},(\alpha \neq 0)$ et $\tau \in \hat{G}$ associée à l'orbite $G \cdot f$. Prenons deux polarisations en $f, \mathfrak{h}_{1}=\operatorname{vect}(A, Y, Z)_{\mathbf{R}}$ et $\mathfrak{h}_{2}=\operatorname{vect}(A, X, Z)_{\mathbf{R}}$ qui vérifient la condition de Pukanszky. Pour la première,

$$
W^{\diamond}=(A+1)(Z+i \alpha)-X Y-i \alpha\left(A+i \beta+\frac{1}{2}\right)+i \alpha\left(i \beta-\frac{1}{2}\right) \equiv i \alpha\left(i \beta-\frac{1}{2}\right)
$$

$\bmod \mathcal{U}(\mathfrak{g}) \mathfrak{a}_{\tau}$. Pour la deuxième

$$
W^{\diamond}=A(Z+i \alpha)-Y X-i \alpha\left(A+i \beta-\frac{1}{2}\right)+i \alpha\left(i \beta-\frac{1}{2}\right) \equiv i \alpha\left(i \beta-\frac{1}{2}\right)
$$

$\bmod \mathcal{U}(\mathrm{g}) \mathfrak{a}_{\tau}$. D'autre part, il est facile de voir que $\mathrm{d} \tau(W) \varphi=i \alpha\left(i \beta-\frac{1}{2}\right) \varphi$ pour tout $\varphi \in \mathcal{H}_{\tau}^{+\infty}$.

Soit $f=\alpha Y^{\star}+\beta X^{\star}\left(\alpha^{2}+\beta^{2} \neq 0\right)$. Ici $\mathfrak{h}=\operatorname{vect}(X, Y, Z)_{\mathbf{R}}$ est une polarisation réelle en $f$ vérifiant la condition de Pukanszky et

$$
W^{\diamond}=A Z-Y(X+i \beta)+i \beta(Y+i \alpha)+\alpha \beta \equiv \alpha \beta
$$

modulo $\mathcal{U}(\mathrm{g}) \mathfrak{a}_{\tau}$. Par ailleurs $\mathrm{d} \tau(W) \varphi=\alpha \beta \varphi$ pour tout $\varphi \in \mathcal{H}_{\tau}^{+\infty}$.

\section{Etude de la Question 2.2}

Ce paragraphe ne concerne que des groupes de Lie nilpotents. Soient donc $G=\exp g$ un groupe de Lie nilpotent d'algèbre de Lie $\mathfrak{g}, H=\exp \mathfrak{h}$ un sous groupe analytique de $G$, ayant l'algèbre de Lie $\mathfrak{h}, f \in \mathfrak{g}^{\star}$ telle que $f([\mathfrak{h}, \mathfrak{h}])=\{0\}$ et $\chi_{f}$ le caractère unitaire de $H$ défini par $\chi_{f}(\exp Y)=\mathrm{e}^{\sqrt{-1} f(Y)}, Y \in \mathfrak{h}$. Soit

$$
\mathcal{S}:\{0\}=\mathfrak{g}_{0} \subset \mathfrak{g}_{1} \subset \cdots \subset \mathfrak{g}_{n-1} \subset \mathfrak{g}_{n}=\mathfrak{g}
$$

un drapeau d'idéaux de $\mathfrak{g}$ tel que $\operatorname{dim} \mathfrak{g}_{k}=k(0 \leqslant k \leqslant n)$. Soit $\mathcal{J}=\left\{i_{1}<i_{2}<\cdots<\right.$ $\left.i_{d}\right\}$ l'ensemble d'indices $i(1 \leqslant i \leqslant n)$ tels que $\mathfrak{h} \cap \mathfrak{g}_{i} \neq \mathfrak{h} \cap \mathfrak{g}_{i-1}$, et posons

$$
\mathcal{I}=\left\{j_{1}<j_{2}<\cdots<j_{p}\right\}=\{1,2, \ldots, n\} \backslash \mathcal{J}(p=\operatorname{dim} \mathfrak{g} / \mathfrak{h}) .
$$


En posant $\mathfrak{f}_{0}=\mathfrak{h}$ et $\mathfrak{f}_{r}=\mathfrak{h}+\mathfrak{g}_{j_{r}}$ pour $1 \leqslant r \leqslant p$, on obtient une suite des sousalgèbres de $\mathrm{g}$ :

$$
\mathfrak{h}=\mathfrak{f}_{0} \subset \mathfrak{f}_{1} \subset \cdots \subset \mathfrak{f}_{p-1} \subset \mathfrak{f}_{p}=\mathfrak{g}, \quad \operatorname{dim}\left(\mathfrak{f}_{r} / \mathfrak{f}_{r-1}\right)=1 .
$$

D'autre part, en considérant $\mathfrak{h}_{s}=\mathfrak{h} \cap \mathfrak{g}_{i},(1 \leqslant s \leqslant d)$, nous procurons un drapeau d'idéaux de $\mathfrak{h}$ :

$$
\{0\}=\mathfrak{h}_{0} \subset \mathfrak{h}_{1} \subset \mathfrak{h}_{2} \subset \cdots \subset \mathfrak{h}_{d-1} \subset \mathfrak{h}_{d}=\mathfrak{h}, \quad \operatorname{dim} \mathfrak{h}_{s}=s, s=0, \ldots, d .
$$

En extrayant un vecteur non-nul $Y_{s} \in \mathfrak{h}_{s} \backslash \mathfrak{h}_{s-1}$ pour $1 \leqslant s \leqslant d$, on forme une base de Malcev $\left\{Y_{1}, \ldots, Y_{d}\right\}$ de $\mathfrak{h}$. De la même manière, en extrayant un vecteur non-nul $X_{r} \in \mathfrak{f}_{r} \backslash \mathfrak{f}_{r-1}$ pour $1 \leqslant r \leqslant p$, on forme une base de Malcev $\left\{X_{1}, \ldots, X_{p}\right\}$ de $g$ relative à $\mathfrak{h}$. Pour la simplicité des notations, on écrit $\mathfrak{h}^{\prime}$ à la place de $\mathfrak{h}_{d-1}$ et construit $\tau^{\prime}=\operatorname{Ind}_{H^{\prime}}^{G} \chi_{f}$, où $H^{\prime}=\exp \mathfrak{h}^{\prime}$, ainsi que $\tau=\operatorname{Ind}_{H}^{G} \chi_{f}$. De même, écrivons $\mathfrak{g}^{\prime}$ au lieu de $\mathfrak{f}_{p-1}$ et posons $G^{\prime}=\exp \mathfrak{g}^{\prime}$.

Nous considérons comme avant l'algèbre $D_{\tau}(G / H)$ des opérateurs différentiels $G$-invariants associée à $\tau$, et l'exprimons comme d'habitude en termes de l'algèbre enveloppante $\mathcal{U}(\mathfrak{g})$ de $\mathfrak{g}_{\mathrm{C}}$.

Cela étant, remarquons d'abord que chaque élément de $D_{\tau}(G / H) \cong \mathcal{U}(\mathfrak{g}, \tau) / \mathcal{U}(\mathfrak{g}) \mathfrak{a}_{\tau}$ admet un unique élément représentatif $W \in \mathcal{U}(\mathrm{g}, \tau)$ qui s'écrit comme une combinaison linéaire des momômes

$$
X^{M}=X_{p}^{m_{p}} X_{p-1}^{m_{p-1}} \cdots X_{2}^{m_{2}} X_{1}^{m_{1}}, M=\left(m_{p}, m_{p-1}, \ldots, m_{2}, m_{1}\right) \in \mathbb{N}^{p},
$$

où $\mathbb{N}=\{0,1,2, \ldots\}$. A savoir,

$$
W=\sum_{M} c_{M} X^{M}\left(c_{M} \in \mathbb{C}\right) .
$$

Cela posé, le lemme suivant est immédiat:

LEMME 4.1. La condition $\left[Y_{s}, W\right] \in \mathcal{U}(\mathfrak{g}) \mathfrak{a}_{\tau},(1 \leqslant s \leqslant d)$ entraine $\left[Y_{s}, W\right] \in \mathcal{U}(\mathfrak{g}) \mathfrak{a}_{\tau^{\prime}}$.

Vis à vis à l'écriture (6), il est immédiat que nous pouvons écrire tout élément $W \in \mathcal{U}(\mathfrak{g}, \tau)$ comme

$$
W=\sum_{k=0}^{m} X_{p}^{k} a_{k}, a_{k} \in \mathcal{U}\left(\mathrm{g}^{\prime}\right), \quad 0 \leqslant k \leqslant m .
$$

Nous allons démontrer que lorsque nous disons qu'il existe $W \in \mathcal{U}(\mathfrak{g}, \tau)$ tel que $W \notin \mathcal{U}\left(\mathfrak{g}^{\prime}\right)$, nous pouvons supposer que $m=1$.

\section{LEMME 4.2. Soit}

$$
W=\sum_{k=0}^{m} X_{p}^{k} a_{k}, \quad m \geqslant 2, \quad a_{k} \in \mathcal{U}\left(\mathfrak{g}^{\prime}\right), \quad 0 \leqslant k \leqslant m .
$$

Si $W \in \mathcal{U}(\mathfrak{g}, \tau)$ et $a_{m} \notin \mathcal{U}(\mathfrak{g}) \mathfrak{a}_{\tau}$, alors il existe un élément $W^{\prime}$ de la forme $W^{\prime}=X_{p} U+V$ appartenant à $\mathcal{U}(\mathfrak{g}, \tau)$ tel que $U, V \in \mathcal{U}\left(\mathfrak{g}^{\prime}\right)$ et $U \notin \mathcal{U}(\mathfrak{g}) \mathfrak{a}_{\tau}$. 
Preuve. Soit $Y \in \mathfrak{h}$, on peut montrer par récurrence que pour tout entier $r \geqslant 1$ on a:

$$
\left[X_{p}^{r}, Y\right]=\sum_{j=1}^{r} X_{p}^{j-1}\left[X_{p}, Y\right] X_{p}^{r-j}
$$

D'où, pour $Y \in \mathfrak{h}$,

$$
\begin{aligned}
{[W, Y]=} & X_{p}^{m}\left[a_{m}, Y\right]+\sum_{j=1}^{m} X_{p}^{j-1}\left[X_{p}, Y\right] X_{p}^{m-j} a_{m}+X_{p}^{m-1}\left[a_{m-1}, Y\right]+ \\
& +\left(\text { termes de degré } \leqslant m-2 \text { par rapport à } X_{p}\right) \\
= & X_{p}^{m}\left[a_{m}, Y\right]+X_{p}^{m-1}\left(m\left[X_{p}, Y\right] a_{m}+\left[a_{m-1}, Y\right]\right)+ \\
& +(\text { termes de degré } \leqslant m-2) \in \mathcal{U}(\mathrm{g}) \mathfrak{a}_{\tau} .
\end{aligned}
$$

Il en résulte que $a_{m} \in \mathcal{U}(\mathfrak{g}, \tau)$ et que $m X_{p} a_{m}+a_{m-1} \in \mathcal{U}(\mathfrak{g}, \tau)$.

Nous allons essayer le premier pas vers l'assertion (2.4). Soit $\tau^{\prime \prime}=\operatorname{Ind}_{H^{\prime}}^{G^{\prime}} \chi_{f}$ et supposons que

$$
\mathcal{U}\left(\mathfrak{g}, \tau^{\prime}\right) \not \subset \mathcal{U}\left(\mathfrak{g}^{\prime}\right)+\mathcal{U}(\mathfrak{g}) \mathfrak{a}_{\tau^{\prime}}
$$

ce qui entaîne que les $H^{\prime}$-orbites génériques dans $\Gamma_{\tau^{\prime}}=f+\left(\mathfrak{G}^{\prime}\right)^{\perp}$ sont non-saturées par rapport à $\mathfrak{g}^{\prime}$ si l'assertion (2.4) était établie pour les donnés $\mathfrak{g}, \mathfrak{h}^{\prime}$ et $f$. Si on voudrait engager un raisonnement de récurrence, alors cette première supposition correspond bien à l'hypothèse de cette récurrence. Supposons encore que $\mathcal{U}\left(\mathfrak{g}^{\prime}, \tau^{\prime \prime}\right) \not \subset \mathcal{U}(\mathfrak{g}, \tau)$. L'assertion (2.4) établie pour les donnés $\mathfrak{g}, \mathfrak{h}^{\prime}$ et $f$, cette deuxième supposition signifirait que l'élément $Y_{d}$ de $\mathfrak{h}$ trouve dans $\mathfrak{g}^{\prime}$ son partenaire relative à la structure symplectique induite par la forme bilinéaire $B_{l}, B_{l}(X, Y)=$ $l([X, Y])(X, Y \in \mathfrak{g})$ pour $l \in \Gamma_{\tau}$ générique. Cela entraîne par suite que les $H$-orbites génériques dans $\Gamma_{\tau}$ sont de même non-saturées par rapport à $\mathfrak{g}^{\prime}$.

Dans cette situation, nous nous proposons de montrer que $\mathcal{U}(\mathfrak{g}, \tau) \not \subset \mathcal{U}\left(\mathfrak{g}^{\prime}\right)+$ $\mathcal{U}(\mathfrak{g}) \mathfrak{a}_{\tau}$, en construisant de force un nouveau élément voulu.

D'après la première hypothèse, il existe $W^{\prime} \in \mathcal{U}\left(\mathfrak{g}, \tau^{\prime}\right)$ s'écrivant $W^{\prime}=X_{p} U^{\prime}+V^{\prime}$ avec $U^{\prime}, V^{\prime} \in \mathcal{U}\left(\mathfrak{g}^{\prime}\right)$ vérifiant $U^{\prime} \notin \mathcal{U}(\mathfrak{g}) \mathfrak{a}_{\tau^{\prime}}$.

Si $U^{\prime} \in \mathcal{U}(\mathfrak{g}) \mathfrak{a}_{\tau}$, on écrit uniquement modulo $\mathcal{U}(\mathfrak{g}) \mathfrak{a}_{\tau^{\prime}}$

$$
\begin{aligned}
U^{\prime} & =\sum_{q \geqslant 1} \sum_{\bar{M}_{q}} c_{\bar{M}_{q}} X_{q}^{\bar{M}}\left(Y_{d}+\sqrt{-1} f\left(Y_{d}\right)\right)^{q}=U^{\prime \prime}\left(Y_{d}+\sqrt{-1} f\left(Y_{d}\right)\right), \\
V^{\prime} & =\sum_{t \geqslant 0} \sum_{\bar{M}_{t}} c_{\bar{M}}^{\prime} X^{\bar{M}}\left(Y_{d}+\sqrt{-1} f\left(Y_{d}\right)\right)^{t} .
\end{aligned}
$$

D'où

$$
W^{\prime}=W^{\prime \prime}\left(Y_{d}+\sqrt{-1} f\left(Y_{d}\right)\right)+\sum_{\bar{M}_{0}} c_{\bar{M}_{0}}^{\prime} X_{0}^{\bar{M}}\left(\text { modulo } \mathcal{U}(\mathfrak{g}) \mathfrak{a}_{\tau^{\prime}}\right)
$$


avec

$$
W^{\prime \prime}=X_{p} U^{\prime \prime}+\sum_{t \geqslant 1} \sum_{\bar{M}_{t}} c_{\bar{M}_{t}}^{\prime} X^{\bar{M}_{t}}\left(Y_{d}+\sqrt{-1} f\left(Y_{d}\right)\right)^{t-1} .
$$

Pour $1 \leqslant s \leqslant d-1$, nous avons que:

$$
\left[Y_{s}, W^{\prime}\right] \in \mathcal{U}(\mathfrak{g}) \mathfrak{a}_{\tau^{\prime}} .
$$

Nous allons prouver que (8) entraîne que $\left[Y_{s}, W^{\prime \prime}\right] \in \mathcal{U}(\mathfrak{g}) \mathfrak{a}_{\tau^{\prime}}$, c'est à dire que $W^{\prime \prime} \in \mathcal{U}\left(\mathfrak{g}, \tau^{\prime}\right)$. En effet, notons

$$
T_{0}=\sum_{\bar{M}_{0}} c_{\bar{M}_{0}}^{\prime} X^{\bar{M}_{0}} .
$$

Pour $1 \leqslant s \leqslant d-1$, nous avons que

$$
\left[W^{\prime}, Y_{s}\right]=W^{\prime \prime}\left[Y_{d}, Y_{s}\right]+\left[W^{\prime \prime}, Y_{s}\right]\left(Y_{d}+\sqrt{-1} f\left(Y_{d}\right)\right)+\left[T_{0}, Y_{s}\right] .
$$

Cela implique aussitôt que $\left[T_{0}, Y_{s}\right] \in \mathcal{U}(\mathfrak{g}) \mathfrak{a}_{\tau^{\prime}}$ et donc $T_{0} \in \mathcal{U}\left(\mathrm{g}, \tau^{\prime}\right)$. Nous affirmons maintenant que $\left[W^{\prime \prime}, Y_{s}\right] \in \mathcal{U}(\mathrm{g}) \mathfrak{a}_{\tau^{\prime}}$. Pour cela, posons $U_{s}=Y_{s}+\operatorname{if}\left(Y_{s}\right), 1 \leqslant s \leqslant d$. Nous allons nous servir de l'implication suivante: Soit $W \in \mathcal{U}(\mathfrak{g})$ telle que $W U_{d} \in \mathcal{U}(\mathrm{g}) \mathfrak{a}_{\tau^{\prime}}$, alors $W$ est lui même dans $\mathcal{U}(\mathrm{g}) \mathfrak{a}_{\tau^{\prime}}$. En effet, écrivons $W$ comme une somme de monôncs de base $X^{\alpha} U_{d}^{k} U_{0}^{\beta}$ avec $U_{0}^{\beta}=U_{d-1}^{\beta_{d-1}} \cdots U_{1}^{\beta_{1}}$,

$$
W=\sum_{\alpha, k, \beta} C_{\alpha, k, \beta} X^{\alpha} U_{d}^{k} U_{0}^{\beta} .
$$

Remarquons que $\left[U_{0}^{\beta}, U_{d}\right] \in \sum_{\beta^{\prime}} \mathrm{C} U_{0}^{\beta^{\prime}} \subset \mathcal{U}(\mathrm{g}) \mathfrak{a}_{\tau^{\prime}}$ pour tout multi-indice $\beta$. Ainsi, en utilisant le fait que $W U_{d} \in \mathcal{U}(\mathrm{g}) \mathfrak{a}_{\tau^{\prime}}$, on aura

$$
\sum_{\alpha, k, \beta} C_{\alpha, k, \beta} X^{\alpha} U_{d}^{k+1} U_{0}^{\beta} \in \mathcal{U}(\mathrm{g}) \mathfrak{a}_{\tau^{\prime}}
$$

et donc que $C_{\alpha, k, 0}=0$. Ainsi

$$
W=\sum_{\alpha, k, \beta \neq 0} C_{\alpha, k, \beta} X^{\alpha} U_{d}^{k} U_{0}^{\beta} \in \mathcal{U}(\mathfrak{g}) \mathfrak{a}_{\tau^{\prime}},
$$

ce qui termine la preuve de l'implication.

En répétant ces manipulations si besoin est, on peut supposer dès le début que $U^{\prime} \notin \mathcal{U}(\mathfrak{g}) \mathfrak{a}_{\tau}$. Ou plus encore nous pouvons prendre $W^{\prime}$ de telle façon que $U^{\prime}, V^{\prime}$ s'écrivent dans une combinaison linéaire de $X^{\bar{M}}=X_{p-1}^{m_{p-1}} \cdots X_{1}^{m_{1}}$.

Appliquons à plusieurs reprises ad $Y_{d}$ à $W^{\prime}$ jusqu'à ce qu'on ait $\left(\operatorname{ad} Y_{d}\right)^{m}$ $\left(W^{\prime}\right) \in \mathcal{U}(\mathfrak{g}) \mathfrak{a}_{\tau}$ pour la première fois. D'ailleurs, en remplaçant $W^{\prime} \operatorname{par}\left(\operatorname{ad} Y_{d}\right)^{m^{\prime}}$ $\left(W^{\prime}\right)$ avec le plus grand exposant $m^{\prime}$ vérifiant:

$\left(\operatorname{ad} Y_{d}\right)^{m^{\prime}}\left(W^{\prime}\right) \notin \mathcal{U}\left(\mathfrak{g}^{\prime}\right)$ (modulo $\left.\mathcal{U}(\mathfrak{g}) \mathfrak{a}_{\tau}\right)$

qui, bien évidemment, appartient à $\mathcal{U}\left(\mathfrak{g}, \tau^{\prime}\right)$ et il s'écrit sous une forme (4), nous pouvons supposer que ad $Y_{d}\left(W^{\prime}\right) \in \mathcal{U}\left(\mathfrak{g}^{\prime}\right)$ modulo $\mathcal{U}(\mathfrak{g}) \mathfrak{a}_{\tau}$.

Si $m=1, W^{\prime} \in \mathcal{U}(\mathfrak{g}, \tau)$ et il ne nous reste plus rien à faire. 
Supposons donc que $m \geqslant 2$. Pour $j \in \mathbb{N}^{\star}$ arbitraire, on voit aussitôt que

$\left(\operatorname{ad} Y_{d}\right)^{j}\left(W^{\prime}\right) \in \mathcal{U}\left(\mathfrak{g}, \tau^{\prime}\right) \cap \mathcal{U}\left(\mathrm{g}^{\prime}\right)$

modulo $\mathcal{U}(\mathfrak{g}) \mathfrak{a}_{\tau^{\prime}}$, d'où en particulier $U^{\prime} \in \mathcal{U}\left(\mathfrak{g}, \tau^{\prime}\right) \cap \mathcal{U}\left(\mathfrak{g}^{\prime}\right)=\mathcal{U}\left(\mathfrak{g}^{\prime}, \tau^{\prime \prime}\right)$. Et nous avons remarqué d'après le lemme (4.1) qu'on peut choisir $W^{\prime}$ de sorte que $\left(\operatorname{ad} Y_{d}\right)^{j}\left(W^{\prime}\right) \in \mathcal{U}(\mathrm{g}) \mathfrak{a}_{\tau}$ si et seulement si $\left(\operatorname{ad} Y_{d}\right)^{j}\left(W^{\prime}\right) \in \mathcal{U}(\mathrm{g}) \mathfrak{a}_{\tau^{\prime}}$. Afin d'alléger les notations, nous posons

$$
e_{j}=\left(\operatorname{ad} Y_{d}\right)^{j-1}\left(W^{\prime}\right)(1 \leqslant j \leqslant m)
$$

en convenant que $e_{1}=W^{\prime}$.

Supposons encore que $m \geqslant 3$. Alors on peut supposer que $m=2 q+1$ est impair, sinon on remplacerait $W^{\prime}$ par $W^{\prime}\left(e_{m-1}+c e_{m}\right)(0 \neq c \in \mathbb{C})$.

LEMME 4.3. Soit

$$
\begin{aligned}
W= & \left(e_{1} e_{m}+e_{m} e_{1}\right)-\left(e_{2} e_{m-1}+e_{m-1} e_{2}\right)+\cdots+(-1)^{q-2}\left(e_{q-1} e_{q+3}+e_{q+3} e_{q-1}\right)+ \\
& +(-1)^{q-1}\left(e_{q} e_{q+2}+e_{q+2} e_{q}\right)+(-1)^{q} e_{q+1}^{2} .
\end{aligned}
$$

Alors $\left[Y_{d}, W\right]=e_{1} e_{m+1}+e_{m+1} e_{1} \in \mathcal{U}(\mathrm{g}) \mathfrak{a}_{\tau}$ et par conséquent que $W$, qui s'écrit dans une forme (4), appartient à $\mathcal{U}(\mathfrak{g}, \tau)$.

Preuve. Nous écrivons

$$
W=\sum_{j=1}^{q}(-1)^{j-1}\left(e_{j} e_{m+1-j}+e_{m+1-j} e_{j}\right)+(-1)^{q} e_{q+1}^{2} .
$$

Ensuite,

$$
\begin{aligned}
{\left[Y_{d}, W\right]=} & \sum_{j=1}^{q}(-1)^{j-1}\left(e_{j+1} e_{m+1-j}+e_{m+1-j} e_{j+1}\right)+(-1)^{j-1}\left(e_{j} e_{m+2-j}+e_{m+2-j} e_{j}\right)+ \\
& \quad+(-1)^{q}\left(e_{q+1} e_{q+2}+e_{q+2} e_{q+1}\right) \\
= & e_{1} e_{m+1}+e_{m+1} e_{1} .
\end{aligned}
$$

Maintenant, nous savons que $e_{m+1}=\left[Y_{d}, e_{m}\right] \in \mathcal{U}(\mathfrak{g}) \mathfrak{a}_{\tau}$ et donc d'après le procédé décrit au début de ce paragraphe, $\left[Y_{d}, e_{m}\right] \in \mathcal{U}(\mathfrak{g}) \mathfrak{a}_{\tau^{\prime}}$. Ainsi, $e_{m+1} e_{1} \in \mathcal{U}(\mathfrak{g}) \mathfrak{a}_{\tau^{\prime}} \subset \mathcal{U}(\mathfrak{g}) \mathfrak{a}_{\tau}$.

Dans le cas éventuel où l'on doit remplacer $e_{1}=W^{\prime} \operatorname{par} \tilde{e}=W^{\prime}\left(e_{m-1}+c e_{m}\right)$, on voit modulo $\mathcal{U}(\mathrm{g}) \mathfrak{a}_{\tau}$, en posant $\tilde{e}_{j}=\left(\operatorname{ad} Y_{d}\right)^{j-1}(\tilde{e})(1 \leqslant j \leqslant m+1)$, que

$$
\tilde{e}_{2} \equiv e_{2}\left(e_{m-1}+c e_{m}\right)+e_{1} e_{m}, \ldots, \tilde{e}_{m} \equiv e_{m}\left(e_{m-1}+c e_{m}\right)+(m-1) e_{m-1} e_{m}, \tilde{e}_{m+1} \equiv m e_{m}^{2} .
$$

D'où notre nouveau élément de $\mathcal{U}(\mathfrak{g}, \tau)$ s'obtient par:

$$
\begin{aligned}
\tilde{W}= & m\left\{e_{1}\left(e_{m-1}+c e_{m}\right) e_{m}^{2}+e_{m}^{2} e_{1}\left(e_{m-1}+c e_{m}\right)\right\}-e_{1} e_{m}\left\{e_{m}\left(e_{m-1}+c e_{m}\right)+\right. \\
& \left.+(m-1) e_{m-1} e_{m}\right\}-\left\{e_{m}\left(e_{m-1}+c e_{m}\right)+(m-1) e_{m-1} e_{m}\right\} e_{1} e_{m}+\tilde{V}
\end{aligned}
$$


avec un certain $\tilde{V} \in \mathcal{U}\left(\mathrm{g}^{\prime}\right)$. Comme on a modulo $\mathcal{U}\left(\mathrm{g}^{\prime}\right)$ que:

$$
\begin{aligned}
\tilde{W} \equiv & m\left(e_{1} e_{m-1} e_{m}^{2}+e_{m}^{2} e_{1} e_{m-1}\right)-(m-1)\left(e_{1} e_{m} e_{m-1}+e_{m-1} e_{m} e_{1}\right) e_{m}- \\
& -\left(e_{1} e_{m}^{2} e_{m-1}+e_{m} e_{m-1} e_{1} e_{m}\right)+c(m-1)\left(e_{1} e_{m}^{2}+e_{m}^{2} e_{1}\right) e_{m},
\end{aligned}
$$

on peut visiblement choisir $0 \neq c \in \mathbb{C}$ de sorte qu'on ait $\tilde{W} \notin \mathcal{U}\left(\mathrm{g}^{\prime}\right)$.

Malheureusement, ce procédé ne marche pas lorsque $m=2$. Vu que $\mathcal{U}\left(\mathrm{g}^{\prime}, \tau^{\prime \prime}\right) \not \subset$ $\mathcal{U}(\mathfrak{g}, \tau)$, on peut élever $m$ en multipliant $W^{\prime}$ par un élément $\hat{e} \in \mathcal{U}\left(\mathfrak{g}^{\prime}, \tau^{\prime \prime}\right)$ n'appartenant pas à $\mathcal{U}(\mathfrak{g}, \tau)$. En effet, prenons un tel $\hat{e}$ vérifiant $\hat{e}_{1}=\left[Y_{d}, \hat{e}\right] \notin \mathcal{U}(\mathfrak{g}) \mathfrak{a}_{\tau}$ mais $\hat{e}_{2}=\left[Y_{d},\left[Y_{d}, \hat{e}\right]\right] \in \mathcal{U}(\mathfrak{g}) \mathfrak{a}_{\tau}$. Le changement de $e_{1}$ à $\tilde{e}_{1}=e_{1} \hat{e}$ nous donne

$$
\tilde{e}_{2}=\left[Y_{d}, \tilde{e}_{1}\right]=e_{2} \hat{e}+e_{1} \hat{e}_{1}, \tilde{e}_{3}=\left[Y_{d},\left[Y_{d}, \tilde{e}_{1}\right]\right] \equiv 2 e_{2} \hat{e}_{1}
$$

modulo $\mathcal{U}(\mathfrak{g}) \mathfrak{a}_{\tau}$.

Si nous formons $\tilde{W}=\left(\tilde{e}_{1} \tilde{e}_{3}+\tilde{e}_{3} \tilde{e}_{1}\right)-\tilde{e}_{2}^{2}$, les deux premiers termes sont de degré 1 par rapport à $X_{p}$ mais le dernier est de degré 2 . Cela va donc nous fournir un élément cherché.

En résumant ce qu'on vient de voir, on obtient le théorème suivant. Soient $H_{s}=\exp \left(\mathfrak{G}_{s}\right)$ et $\tau_{s}=\operatorname{Ind}_{H_{s}}^{G^{\prime}} \chi_{f}$ pour $0 \leqslant s \leqslant d$. En particulier, $\tau_{d-1}=\tau^{\prime \prime}$ et $\tau_{0}$ est la représentation régulière de $G^{\prime}$.

THÉORÈME 4.4. Soit toujours $\mathfrak{h}$ une sous-algèbre de g supposée nilpotente. Si avec les mêmes notations (7) est vérifié et que $\mathcal{U}\left(\mathrm{g}^{\prime}, \tau_{d-1}\right) \not \subset \mathcal{U}\left(\mathrm{g}^{\prime}, \tau_{d}\right)$, alors

$$
\mathcal{U}(\mathfrak{g}, \tau) \not \subset \mathcal{U}\left(\mathfrak{g}^{\prime}\right)+\mathcal{U}(\mathfrak{g}) \mathfrak{a}_{\tau} .
$$

Dans la suite, nous allons utiliser le résultat suivant:

THÉORÈME 4.5 ([18, 19]). Soient $\mathfrak{g}$ une algèbre de Lie nilpotente et $\mathfrak{h}$ une sousalgèbre de $\mathfrak{g}$. Soit $\mathfrak{g}_{0}$ un idéal de codimension 1 dans g contenant $\mathfrak{h}$ telle que $\rho_{0}=$ $\operatorname{Ind}_{H}^{G_{0}} \chi_{f}, G_{0}=\exp \left(\mathrm{g}_{0}\right)$ soit à multiplicités finies. Supposons que $\mathcal{U}(\mathrm{g}, \tau) \not \subset \mathcal{U}\left(\mathrm{g}_{0}\right)+$ $\mathcal{U}(\mathfrak{g}) \mathfrak{a}_{\tau}$, et soit $W$ appartenant à $\mathcal{U}(\mathfrak{g}, \tau)$ de la forme $W=X_{p} U+V$ avec $U, V \in \mathcal{U}\left(\mathfrak{g}_{0}\right)$ vérifiant $U \notin \mathcal{U}(\mathfrak{g}) \mathfrak{a}_{\tau}$. Alors, si $\tau$ est à multiplicités infinies, il existe $T \in \mathcal{U}\left(\mathfrak{g}_{0}, \rho_{0}\right)$ tel que $[T, W] \notin \mathcal{U}(\mathrm{g}) \mathfrak{a}_{\tau}$ et donc l'algèbre $D_{\tau}(G / H)$ est non-commutative.

Comme résultat immédiat du théorème (4.4), nous avons le résultat suivant:

PROPOSITION 4.6. Supposons que g est une algèbre de Lie nilpotente et $\mathfrak{h}$ une sousalgèbre de g. Si $\mathcal{U}\left(\mathfrak{g}^{\prime}\right)=\mathcal{U}\left(\mathfrak{g}^{\prime}, \tau_{0}\right) \not \subset \mathcal{U}\left(\mathfrak{g}^{\prime}, \tau_{1}\right) \not \subset \mathcal{U}\left(\mathfrak{g}^{\prime}, \tau_{2}\right) \not \subset \cdots \not \subset \mathcal{U}\left(\mathfrak{g}^{\prime}, \tau_{d}\right)$, alors il existe un élément $W$ appartenant à $\mathcal{U}(\mathfrak{g}, \tau)$ de la forme $W=X_{p} U+V$ avec $U, V \in \mathcal{U}\left(\mathrm{g}^{\prime}\right)$ vérifiant $U \notin \mathcal{U}(\mathfrak{g}) \mathfrak{a}_{\tau}$. Si de plus $\tau$ est à multiplicités infinies et que $\tau_{d}$ est à multiplicités finies, alors l'algèbre $D_{\tau}(G / H)$ est non-commutative.

Preuve. Pour la première partie, nous utilisons une récurrence sur la dimension de $\mathfrak{h}$. Certainement notre hypothèse est vérifiée pour $\mathfrak{h}_{d-1}$, ainsi (7) est vérifié et le théorème (4.4) nous fournit le résultat cherché. 
Pour la seconde partie, nous utilisons le théorème (4.5).

Nous arrivons maintenant à prouver le théorème suivant:

THÉORÈME 4.7. Supposons que $\mathfrak{g}$ est une algèbre de Lie nilpotente et $\mathfrak{h}$ une sousalgèbre de g. Si $\operatorname{dim} \mathfrak{h} \leqslant 2$, alors l'algèbre $D_{\tau}(G / H)$ est commutative si et seulement si $\tau$ est à multiplicités finies.

Preuve. Rappelons tout d'abord le fait suivant: Soit $(\mathfrak{g}, \mathfrak{h})$ un couple réductif, il existe donc un supplémentaire $\mathfrak{m}$ de $\mathfrak{h}$ dans $\mathfrak{g}$ tel que $[\mathfrak{h}, \mathfrak{m}] \subset \mathfrak{m}$. Soit $\mathfrak{g}_{0}$ un idéal de codimension un contenant $\mathfrak{h}$ et $G_{0}=\exp \left(\mathfrak{g}_{0}\right)$. Supposons que génériquement, les $H$-orbites dans $\mathfrak{m}^{\star}$ sont non-saturées par rapport à $\mathfrak{g}_{0}$. Alors, il est bien connu (cf. [7], page 702) que la symétrisation $\sigma$ est un isomorphisme entre $S(\mathfrak{m})^{H}$ et $\mathcal{U}^{H}(\mathrm{~g}) / \mathcal{U}^{H}(\mathfrak{g}) \cap \mathcal{U}(\mathfrak{g}) \mathfrak{a}_{\tau}$. Si $P$ est un polynôme $H$-invariant qui n'appartient pas à $S\left(\mathfrak{m} \cap \mathfrak{g}_{0}\right)^{H}$, alors $\sigma(P)$ est un élément non-nul de $\mathcal{U}(\mathfrak{g}, \tau)$ vérifiant $\sigma(P) \notin$ $\mathcal{U}\left(\mathfrak{g}_{0}\right)+\mathcal{U}(\mathfrak{g}) \mathfrak{a}_{\tau}$.

Le cas où $\operatorname{dim} \mathfrak{h}=1$ est un exemple où la paire $(\mathfrak{g}, \mathfrak{h})$ est réductive, ce cas a été étudié par Fujiwara, Lion et Mehdi dans [18]. Nous allons scruter donc le cas où $\operatorname{dim} \mathfrak{h}=2$. Nous allons supposer de plus que $\tau$ est à multiplicités infinies et que $\tau_{d}$ est à multiplicités finies. Il est déjà connu que dans la plupart des cas, il existe $W \in \mathcal{U}(\mathfrak{g}, \tau)$ de la forme $W=X_{p} U+V$ avec $U, V \in \mathcal{U}\left(\mathrm{g}^{\prime}\right)$ vérifiant $U \notin \mathcal{U}(\mathrm{g}) \mathfrak{a}_{\tau}$, ce qui entraîne que l'algèbre $D_{\tau}(G / H)$ est non-commutative d'après le théorème (4.5). Nous allons vérifier ce résultat pour tous les cas.

Comme la paire $\left(\mathfrak{g}, \mathfrak{h}_{1}\right)$ est réductive, on constate d'après le rappel fait au début de cette preuve que $\mathcal{U}\left(\mathfrak{g}, \tau^{\prime}\right) \not \subset \mathcal{U}\left(\mathfrak{g}^{\prime}\right)+\mathcal{U}(\mathfrak{g}) \mathfrak{a}_{\tau^{\prime}}$. Si $\mathcal{U}\left(\mathfrak{g}^{\prime}, \tau_{1}\right) \not \subset \mathcal{U}\left(\mathfrak{g}^{\prime}, \tau_{2}\right)$, alors c'est le théorème (4.4) qui nous donne immédiatement la réponse. Supposons donc que

$$
\mathcal{U}\left(\mathrm{g}^{\prime}, \tau_{1}\right) \subset \mathcal{U}\left(\mathrm{g}^{\prime}, \tau_{2}\right)
$$

et remarquons que cette condition entraîne la finitude des multiplicités de $\tau_{1}$. En effet, rappelons la définition

$$
\Gamma_{j}=\left\{l \in \mathfrak{f}_{j}^{\star} \mid l(Y)=f(Y), Y \in \mathfrak{h}\right\}
$$

et introduisons

$$
\Gamma_{j}^{1}=\left\{l \in \mathfrak{f}_{j}^{\star} \mid l(Y)=f(Y), Y \in \mathfrak{h}_{1}\right\} .
$$

Si au passage de $\mathfrak{f}_{j-1}$ à $\mathfrak{f}_{j}$, $(1 \leqslant j \leqslant p-1)$ presque toutes les $H_{1}$-orbites dans $\Gamma_{j}^{1}$ sont non-saturées par rapport à $\mathfrak{f}_{j-1}$, on trouve $W_{j} \in \mathcal{U}\left(\mathfrak{g}^{\prime}, \tau_{1}\right) \cap \mathcal{U}\left(\mathfrak{f}_{j}\right)$ tel que $W_{j} \notin \mathcal{U}\left(\mathfrak{f}_{j-1}\right)+\mathcal{U}\left(\mathfrak{f}_{j}\right) \mathfrak{a}_{\tau_{1}}$. D'après ce que nous avons vu dans les détails du théorème (4.4), nous pouvons modifier ce $W_{j}$ de sorte que nous ayons $W_{j} \notin \mathcal{U}\left(\mathfrak{f}_{j-1}\right)+\mathcal{U}\left(\mathfrak{f}_{j}\right) \mathfrak{a}_{\tau}$. S'il en est ainsi, l'hypothèse $\mathcal{U}\left(\mathrm{g}^{\prime}, \tau_{1}\right) \subset \mathcal{U}\left(\mathrm{g}^{\prime}, \tau_{2}\right)$ assure que $W_{j} \in \mathcal{U}(\mathrm{g}, \tau)$ et finalement presque toutes les $K_{j}$-orbites, $K_{j}=\exp \left(\mathfrak{f}_{j}\right)$, rencontrant $\Gamma_{j}$ sont non-saturées par rapport à $\mathfrak{j}_{j-1}$. En continuant ces observations, nous arrivons à la conclusion que $\tau_{1}$ est à multiplicités finies comme $\tau_{2}$ l'est. Cela signifie que

$$
\mathfrak{h}=\mathfrak{h}_{2} \subset \mathfrak{h}_{1}+\mathfrak{f}_{p-1}(l)
$$


pour presque toutes les $l \in \Gamma_{p-1}$. Comme presque partout dans $\Gamma_{\tau}=\Gamma_{p}$, les $H$-orbites sont non-saturées par rapport à $\mathfrak{f}_{p-1}$, il vient que

$$
\mathfrak{h}=\mathfrak{h}_{2} \subset \mathfrak{h}_{1}+\mathfrak{g}(l)
$$

presque partout dans $\Gamma_{\tau}$ relativement à la mesure de Lebesgue dans $\Gamma_{\tau}$.

Conformément au théorème (4.5), nous allons prouver que nous pouvons produire un élément $X \in \mathcal{U}(\mathfrak{g}, \tau)$ qui n'est pas dans $\mathcal{U}\left(\mathfrak{g}^{\prime}\right)+\mathcal{U}(\mathfrak{g}) \mathfrak{a}_{\tau}$.

Soit $T \in \mathfrak{g}$ tel que $\left[T, Y_{1}\right]=0$. Alors $\left[T, Y_{2}\right] \in \mathfrak{h}_{1} \cap \operatorname{ker} f$ d'après (9). Donc si $T \notin \mathfrak{g}^{\prime}$, un tel $T \in \mathfrak{g}$ existe pourvu que $\left[X_{p}, Y_{1}\right] \in\left[\mathfrak{g}^{\prime}, Y_{1}\right]$, il ne nous reste rien à faire. Soit $j_{0}$ le plus petit indice vérifiant $\left[X_{j_{0}}, Y_{1}\right] \neq 0$. Si $j_{0}=p$, alors $\left[\mathfrak{g}^{\prime}, Y_{1}\right]=0$ et notre hypothèse sur les multiplicités entraîne que $\left[X_{p}, Y_{1}\right]=0$, ce qui achève le but. Soit $j_{0} \leqslant p-1$,

$$
Y_{2}-\frac{l\left(\left[X_{j_{0}}, Y_{2}\right]\right)}{l\left(\left[X_{j_{0}}, Y_{1}\right]\right)} Y_{1} \in \mathrm{g}(l)
$$

pour presque toutes les $l \in \Gamma_{\tau}$. D'où

$$
l\left(\left[X_{j_{0}}, Y_{1}\right]\right) l\left(\left[X_{p}, Y_{2}\right]\right)=l\left(\left[X_{p}, Y_{1}\right]\right) l\left(\left[X_{j_{0}}, Y_{2}\right]\right)
$$

pour presque toutes les $l \in \Gamma_{\tau}$. Ici $\left[X_{j_{0}}, Y_{1}\right]$ et $\left[X_{p}, Y_{1}\right]$ sont linéairement indépendants, et en modifiant $Y_{2}$, on peut supposer que $l\left(\left[X_{p}, Y_{2}\right]\right)$ est constante lorsque $\left[X_{p}, Y_{2}\right]$ et $\left[X_{p}, Y_{1}\right]$ sont linéairement dépendants modulo $\mathfrak{h}_{1}$. De même lorsque $\left[X_{p}, Y_{2}\right]$ est colinéaire à $\left[X_{j_{0}}, Y_{2}\right]$ après une addition éventuelle à $X_{p}$ d'un multiple de $X_{j_{0}}$. Tout compte fait, nous sommes amenés à la condition $\left[X_{j_{0}}, Y_{2}\right] \in \mathfrak{h}_{1} \cap \operatorname{ker} f$ et $\left[X_{p}, Y_{2}\right] \in \mathfrak{h}_{1} \cap \operatorname{ker} f$. S'il en est ainsi, la formule (10) nous amène au fait que $Y_{2} \in \mathfrak{g}(l)$ pour presque toutes les $l \in \Gamma_{\tau}$. Cela implique que $\left[X, Y_{2}\right] \in \mathfrak{h}_{1} \cap \operatorname{ker} f$ pour tout $X \in \mathfrak{g}$. En particulier, [[g g, $\left.\mathfrak{g}], Y_{2}\right]=\{0\}$. Si $Y_{2}$ est central dans $\mathfrak{g}$, rien à faire encore. Sinon, $f\left(Y_{1}\right)=0$, et l'élément $X_{p}$ nous convient si $\left[X_{p}, Y_{2}\right]=0$. En effet, il existe $X \in \mathfrak{g}$ tel que $Y_{1}=\left[X, Y_{2}\right]$ et

$$
\left[X_{p}, Y_{1}\right]=\left[X_{p},\left[X, Y_{2}\right]\right]=\left[\left[X_{p}, X\right], Y_{2}\right]+\left[X,\left[X_{p}, Y_{2}\right]\right]=0 .
$$

Enfin, si $\left[X_{p}, Y_{2}\right]=Y_{1}$, alors on a

$$
\left[\left[X_{p}, Y_{1}\right], Y_{1}\right]=\left[\left[X_{p}, Y_{1}\right],\left[X_{p}, Y_{2}\right]\right]=\left[\left[\left[X_{p}, Y_{1}\right], X_{p}\right], Y_{2}\right]+\left[X_{p},\left[\left[X_{p}, Y_{1}\right], Y_{2}\right]\right]=0
$$

compte tenu de $\left[[\mathfrak{g}, \mathfrak{g}], Y_{2}\right]=\{0\}$. Alors nous pouvons aisément constater que l'élément $X_{p}\left[X_{j_{0}}, Y_{1}\right]-\left[X_{p}, Y_{1}\right] X_{j_{0}}$ nous convient, d'où donc le résultat.

Bien qu'il reste essentiellement là, nous énonçons ce résultat sous la forme suivante.

PROPOSITION 4.8. Soient $\mathfrak{g}$ une algèbre de Lie nilpotente et $\mathfrak{h}$ une sous-algèbre de $\mathfrak{g}$.

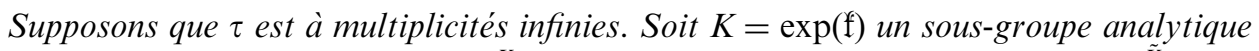
de G contenant $H$ tel que $\tau^{\prime}=\operatorname{Ind}_{H}^{K} \chi_{f}$ soit à multiplicités infinies et que $\tilde{\tau}=\operatorname{Ind}_{H}^{\tilde{K}} \chi_{f}$ soit à multiplicités finies pour tous les sous-groupes analytiques $\tilde{K}$ de $K$ contenant $H$ 
et vérifiant $\operatorname{dim} K / \tilde{K}=1$. S'il existe dans $\mathfrak{h}$ un idéal a de $\mathfrak{f}$ tel que $\operatorname{dim} \mathfrak{h} / \mathfrak{a} \leqslant 2$, alors l'algèbre $D_{\tau}(G / H)$ est non-commutative.

Preuve. Il est évident que nous pouvons supposer que $G=K$. Si h h est elle-même un idéal de $\mathfrak{g}$, le résultat est connu d'après Baklouti et Ludwig (voir [3]). Si dim $\mathfrak{h} / \mathfrak{a}=1$, on peut supposer que $\mathfrak{a}=\mathfrak{h}_{d-1}$ par usage des notations précédentes. Les $H$-orbites génériques dans $\Gamma_{\tau}$ étant non-saturées, $X_{p}$ peut être choisi de sorte qu'on ait $\left[X_{p}, \mathfrak{h}_{d-1}\right] \subset \mathfrak{h}_{d-1} \cap \operatorname{ker} f$. Dans le cas où $\mathfrak{h} \subset \mathfrak{h}_{d-1}+\mathfrak{g}(l)$ pour $l \in \Gamma_{\tau}$ génériques, cela exige que $l\left(\left[X_{p}, \mathfrak{h}\right]\right)=\{0\}$ presque partout dans $\Gamma_{\tau}$ et puis que $\left[X_{p}, \mathfrak{h}\right] \subset \mathfrak{h} \cap \operatorname{ker} f$. En bref, $X_{p}$ nous convient.

Lorsque $\mathfrak{h} \not \subset \mathfrak{h}_{d-1}+\mathfrak{g}(l)$ pour $l \in \Gamma_{\tau}$ génériques, on se trouve d'après les détails du preuve du théorème 4.7 dans le cas où

$$
\mathcal{U}\left(\mathfrak{g}^{\prime}, \tau_{d-1}\right) \not \subset \mathcal{U}\left(\mathfrak{g}^{\prime}, \tau_{d}\right)
$$

pour un certain idéal $\mathfrak{g}^{\prime}$ de codimension 1 dans $\mathfrak{g}$ par abus des notations précédentes. Ces détails sont faits quand $\operatorname{dim} \mathfrak{h}=2$, mais rien ne nous empêche de les appliquer dans notre cas. Nous somme finalement en mesure d'appliquer le théorème 4.4 pour arriver au résultat voulu.

De même, $\mathfrak{a}=\mathfrak{h}_{d-2}$ si $\operatorname{dim} \mathfrak{h} / \mathfrak{a}=2$. Posons

$$
\mathfrak{n}=\{X \in \mathfrak{g} \mid[X, \mathfrak{a}] \subset \mathfrak{a} \cap \operatorname{ker} f\} .
$$

Alors $\mathfrak{n}$ est une sous-algèbre de $\mathfrak{g}$ contenant $\mathfrak{h}$. Les sous-algèbres $\mathfrak{h}_{i}, 1 \leqslant i \leqslant d-2$ étant prises comme des idéaux de $\mathfrak{g}$. Soit $i_{0}$ le premier indice pour lequel $\left[\mathfrak{g}, \mathfrak{h}_{i_{0}}\right] \not \subset$ $\operatorname{ker} f$. Si $\mathfrak{n} \neq \mathfrak{g}$, alors l'indice $i_{0}$ existe. Posons alors

$$
\tilde{\mathfrak{f}}=\left\{X \in \mathfrak{g} \mid\left[X, \mathfrak{h}_{i_{0}}\right] \subset \operatorname{ker} f\right\} .
$$

Alors il est clair que $\tilde{f}$ est un idéal de codimension 1 dans $\mathfrak{g}$ et que $\mathfrak{h} \subset \mathfrak{n} \subset \tilde{\mathfrak{f}}$. Il va de soi de poser $\tilde{K}=\exp (\tilde{\mathfrak{f}})$. Nous affirmons maintenant que $\tilde{\tau}=\operatorname{Ind}_{H}^{\tilde{K}} \chi_{f}$ doit être à multiplicités infinies. En effet, supposons le contraire et remarquons que les $H$-orbites génériques dans $\Gamma_{\tau}$ sont saturées par rapport à f́. Ceci va impliquer d'après la proposition 3.5.1 que $\tau$ lui-même est à multiplicités finies, ce qui est contradictoire à l'hypothèse.

Tout compte fait, nous sommes dans la situation où $\mathfrak{n}=\mathfrak{g}$ et que $\mathfrak{b}=\mathfrak{a} \cap \operatorname{ker} f$ est un idéal de $\mathfrak{g}$. Si $\mathfrak{b} \neq\{0\}$, nous pouvons descendre au quotient $\mathfrak{g} / \mathfrak{b}$ tout en adaptant un raisonnement de récurrence. Si $\mathfrak{b}=\{0\}$ et $\operatorname{dim} \mathfrak{a}=1$ i.e. $d=3$. Comme $[\mathfrak{g}, \mathfrak{a}]=0$, il se trouve que $\mathfrak{h}_{1}$ est contenue dans le centre de $\mathfrak{g}$ et $f$ ne s'annule pas sur $\mathfrak{h}_{1}$. Rappelons qu'il existe dans $\mathcal{U}(\mathfrak{g}) \backslash \mathcal{U}\left(\mathfrak{g}^{\prime}\right)$ un élément $H_{2}$-invariant et remarquons que $\mathfrak{h}$ est abélienne. S'il en est ainsi, il nous suffit d'appliquer le résultat d'existence à $\tilde{\mathfrak{h}}=\mathbf{R} Y_{d} \oplus \mathbf{R} Y_{d-1}$ pour trouver l'élément cherché.

Remarque 4.9. L'algèbre $\mathfrak{g}$ est toujours considérée nilpotente. La deuxième condition $\mathcal{U}\left(\mathfrak{g}^{\prime}, \tau_{d-1}\right) \not \subset \mathcal{U}\left(\mathfrak{g}^{\prime}, \tau_{d}\right)$ du théorème (4.4) est testée le plus simplement possible comme suit. Posons

$$
\mathfrak{D}=\left\{X \in \mathfrak{g}^{\prime} \mid\left[X, \mathfrak{h}_{d-1}\right] \subset \mathfrak{h}_{d-1} \cap \operatorname{ker} f\right\} .
$$


Pour que la condition en question soit requise, il suffit que $\left[Y_{d}, \grave{\triangleright}\right] \not \subset \mathfrak{h}_{d-1} \cap \operatorname{ker} f$. En particulier, si $f$ s'annulle sur $\mathfrak{h}$, il suffit que le normalisateur dans $\mathfrak{g}^{\prime}$ de $\mathfrak{h}$ est strictement contenu dans celui de $\mathfrak{h}^{\prime}=\mathfrak{h}_{d-1}$.

EXEMPLE 4.10. Si presque toutes les $H$-orbites dans $\Gamma_{\tau}$ sont saturées par rapport à $\mathfrak{g}^{\prime}$, le procédé décrit plus haut ne doit pas marcher. L'exemple le plus simple, c'est comme suit. Soit $\mathfrak{n}=\operatorname{vect}(X, Y, Z)_{\mathbf{R}}$ l'algèbre de Heisenberg, i.e. $[X, Y]=Z$. Soient $f=Z^{*}$ et $\mathfrak{h}=\operatorname{vect}(Y, Z)_{\mathbf{R}}$. Cela implique que $\mathfrak{h}$ est une polarisation de $\mathfrak{g}$ en $f$, que $\tau=\operatorname{Ind}_{H}^{G} \chi_{f}$ est irréductible et donc que l'algèbre $D_{\tau}(G / H)$ est triviale. Considérons le drapeau d'idéaux $\mathcal{S}$ de $\mathfrak{g}$ :

$$
\mathcal{S}:\{0\} \subset \mathbf{R} Z \subset \mathbf{R} Y \oplus \mathbf{R} Z \subset \mathfrak{g} .
$$

D'où $\mathfrak{h}_{1}=\mathbf{R} Z, Y_{1}=Z$ et $\mathfrak{h}_{2}=\mathfrak{g}^{\prime}=\mathbf{R} Y \oplus \mathbf{R} Z$ et $Y_{2}=Y$ avec nos notations. Il est immédiat que $X \in \mathcal{U}\left(\mathfrak{g}, \tau^{\prime}\right)$ qui n'appartient pas à $\mathcal{U}\left(\mathrm{g}^{\prime}\right)$. Cependant nous avons des faits $(\operatorname{ad} Y)^{2}(X)=0, \mathcal{U}\left(\mathfrak{g}^{\prime}, \tau_{1}\right)=\mathcal{U}\left(\mathfrak{g}^{\prime}\right)=\mathcal{U}\left(\mathfrak{g}^{\prime}, \tau_{2}\right)$ et $\mathfrak{h} \not \subset \mathfrak{h}_{1}+\mathfrak{g}(l)$ pour n'importe quelle $l \in \Gamma_{\tau}$.

Passons à l'autre extrême et étudions la situation où $\operatorname{dim} \mathfrak{h}=\frac{1}{2}(\operatorname{dim} \mathfrak{g}+$ $\operatorname{dim} g(f))-1$.

EXEMPLE 4.11. Soit $\mathfrak{g}$ une algèbre nilpotente. Supposons que le centre $z$ de $\mathfrak{g}$ est de dimension 1 , soit $z=\mathbf{R} Z$. Soient $f \in \mathfrak{g}^{*}$ telle que $f(Z)=1, Y \in \mathfrak{g}$ tels que $\mathfrak{a}=\operatorname{vect}(Z, Y)_{\mathbf{R}}$ est un idéal de $\mathfrak{g}, \mathfrak{g}_{0}$ le centralisateur dans $\mathfrak{g}$ de $\mathfrak{a}$ et $\mathfrak{b}$ une polarisation qui n'est pas contenue dans $\mathfrak{g}_{0}$. Posons $\mathfrak{h}=\mathfrak{b} \cap \mathfrak{g}_{0}$ et $\mathfrak{b}=\mathbf{R} X+\mathfrak{h}$. Alors il est clair que $\tau=\operatorname{Ind}_{H}^{G} \chi_{f}$ est à multiplicités infinies et que l'algèbre $D_{\tau}(G / H)$ est noncommutative. En effet, $X, Y \in \mathcal{U}(\mathfrak{g}, \tau)$ et $[X, Y]=Z \notin \mathcal{U}(\mathfrak{g}) \mathfrak{a}_{\tau}$.

PROPOSITION 4.12. Soient $\mathfrak{g}$ une algèbre nilpotente et $\mathfrak{h}$ une sous-algèbre de $\mathfrak{g}$ telles que $\operatorname{dim} \mathfrak{h}=\frac{1}{2}(\operatorname{dim} \mathfrak{g}+\operatorname{dim} \mathfrak{g}(f))-1$. Alors l'algèbre $D_{\tau}(G / H)$ est commutative si et seulement si les multiplicités de $\tau$ sont finies.

Preuve. La condition $\operatorname{dim} \mathfrak{h}=\frac{1}{2}(\operatorname{dim} \mathfrak{g}+\operatorname{dim} \mathfrak{g}(f))-1$ signifie que $\operatorname{dim} \mathfrak{h}=$ $\operatorname{dim} \mathfrak{b}-1$ où $\mathfrak{b}$ est une polarisation en $f$. Si le centre $z$ de $\mathfrak{g}$ n'est pas contenu dans $\mathfrak{h}$, alors $\operatorname{dim} \mathfrak{h} /(\mathfrak{h} \cap \mathfrak{z})=1$ et $\mathfrak{b}=\mathfrak{h}+\mathfrak{z}$ est une polarisation commune pour $l \in \Gamma_{\tau}$ génériques. Si $z \subset \mathfrak{h}$ et si $\operatorname{dim} z \geqslant 2$, on peut passer au quotient $\mathfrak{g} /(z \cap \operatorname{ker} f)$ auquel s'applique l'hypothèse de récurrence.

Supposons donc que $z \subset \mathfrak{h}$ et $\operatorname{dim} z=1$. Si $f_{\mathfrak{l}}=0$, on descend au quotient $\mathfrak{g} / \mathfrak{z}$. Nous sommes donc dans la situation habituelle où nous disposons de $X, Y, Z, \mathrm{~g}_{0}$. Soit $G_{0}=\exp \left(\mathfrak{g}_{0}\right)$.

Nous allons démontrer par récurrence que la commutativité de $D_{\tau}(G / H)$ implique la finitude des multiplicités de $\tau$.

Supposons en premier lieu que $\mathfrak{h} \subset \mathfrak{g}_{0}$, que $\tau_{0}=\operatorname{Ind}_{H}^{G_{0}} \chi_{f}$ est à multiplicités finies et que l'algèbre $D_{\tau}(G / H)$ est abélienne. Nous pouvons choisir une base de Malcev 
$\mathcal{B}=\left\{X_{1}, \ldots, X_{p}\right\}, X_{p}=X$ de $\mathfrak{g}$ relative à $\mathfrak{h}$ telle que $\mathcal{B}_{0}=\left\{X_{1}, \ldots, X_{p-1}\right\}$ soit une base de Malcev de $\mathfrak{g}_{0}$ relative à $\mathfrak{h}$. Nous pouvons supposer encore que $(Y+\mathfrak{g}(f)) \cap \mathfrak{h}=\emptyset$, car si $Y+S \in \mathfrak{h}$ pour un certain $S \in \mathfrak{g}(f)$, les $H$-orbites génériques de $\Gamma_{\tau}$ sont saturées par rapport à $\mathfrak{g}_{0}$ et donc les multiplicités de $\tau$ sont finies. Nous pouvons alors prendre $X_{1}=Y$. Montrons que $D_{\tau}(G / H) \simeq \mathrm{C}[Y]$. En effet, remarquons tout d'abord que $D_{\tau}(G / H) \simeq D_{\tau_{0}}\left(G_{0} / H\right)$ car s'il existe un élément $W=X_{p} U+V$ dans $\mathcal{U}(\mathrm{g}, \tau)$ avec certains $U, V$ dans $\mathcal{U}\left(\mathrm{g}_{0}\right)$ et $U \notin \mathcal{U}\left(\mathrm{g}_{0}\right) \mathfrak{a}_{\tau}$, alors l'algèbre en question serait non-commutative. Ensuite, posons $\mathfrak{h}^{\prime}=\mathfrak{h} \oplus \mathbf{R} Y$ et supposons sans perte de généralités que $f(Y)=0$. Comme $\mathfrak{h}^{\prime}$ est une polarisation en $f$, l'algèbre $D_{\tau^{\prime}}\left(G_{0} / H^{\prime}\right)$ est triviale d'après la proposition (3.7) où nous avons supposé que $H^{\prime}=\exp \left(\mathfrak{h}^{\prime}\right)$ et $\tau^{\prime}=\operatorname{Ind}_{H^{\prime}}^{G_{0}} \chi_{f}$.

Soit $W$ un élément de $\mathcal{U}\left(\mathrm{g}_{0}, \tau_{0}\right)$, alors $[W, \mathfrak{h}] \subset \mathcal{U}\left(\mathrm{g}_{0}\right) \mathfrak{a}_{\tau_{0}}$. Comme $[W, Y]=0$, nous avons que

$$
W \in \mathcal{U}\left(\mathrm{g}_{0}, \tau^{\prime}\right)=\mathrm{C} \cdot 1+\mathcal{U}\left(\mathrm{g}_{0}\right) \mathfrak{a}_{\tau^{\prime}}
$$

encore d'après la proposition 3.7. Utilisons encore les monômes

$$
X_{0}^{\alpha} Y^{k} U^{\beta}=X_{p-1}^{\alpha_{p-1}} \cdots X_{1}^{\alpha_{1}} \cdot Y^{k} \cdot U_{d}^{\beta_{d}} \cdots U_{1}^{\beta_{1}}
$$

avec $U_{j}=Y_{j}+\operatorname{if}\left(Y_{j}\right)$. Ainsi $W$ peut se mettre de la forme

$$
W=\lambda I+\sum_{\alpha,(k, \beta) \neq(0,0)} C_{\alpha, k, \beta} X_{0}^{\alpha} Y^{k} U^{\beta}
$$

pour un certain $\lambda \in \mathrm{C}$ et certains coefficients complexes $C_{\alpha, k, \beta}$. Ainsi modulo $\mathcal{U}\left(\mathfrak{g}_{0}\right) \mathfrak{a}_{\tau_{0}}, W$ pourra s'écrire

$$
W=\lambda I+\sum_{\alpha, k} C_{\alpha, k, 0} X_{0}^{\alpha} Y^{k}
$$

Nous affirmons maintenant que $C_{\alpha, k, 0}$ est nul pour tout $\alpha \neq(0, \ldots, 0)$. En effet, si le contraire est vrai, on pose

$$
W^{\prime}=W-\lambda I=\sum_{\alpha, k} C_{\alpha, k, 0} X_{0}^{\alpha} Y^{k} \in \mathcal{U}\left(\mathfrak{g}_{0}, \tau_{0}\right)
$$

Soit $k_{0}=\inf \left\{k, C_{\alpha, k, 0} \neq 0, \alpha \neq(0, \ldots, 0)\right\}$, alors

$$
W^{\prime}-\sum_{k} C_{(0, \ldots, 0), k, 0} Y^{k}=Y^{k_{0}} \sum_{\alpha, k \geqslant k_{0}} C_{\alpha, k, 0} X_{0}^{\alpha} Y^{k-k_{0}}=Y^{k_{0}} W^{\prime \prime} .
$$

En remarquant que pour $s=1, \ldots, d,\left[Y_{s}, W^{\prime}\right]=Y^{k_{0}}\left[Y_{s}, W^{\prime \prime}\right]$, nous arrivons à la conclusion que

$$
W^{\prime \prime}=\sum_{\alpha, k \geqslant k_{0}} C_{\alpha, k, 0} X_{0}^{\alpha} Y^{k-k_{0}} \in \mathcal{U}\left(\mathfrak{g}_{0}, \tau_{0}\right) \subset \mathcal{U}\left(\mathfrak{g}_{0}, \tau^{\prime}\right)
$$

et donc

$$
W^{\prime \prime}=\sum_{\alpha} C_{\alpha, k_{0}, 0} X_{0}^{\alpha}
$$


modulo $\mathcal{U}\left(\mathfrak{g}_{0}\right) \mathfrak{a}_{\tau^{\prime}}$. Finalement, les coefficients $C_{\alpha, k_{0}, 0}$ doivent être nuls, ce qui est impossible.

Nous en déduisons alors que $D_{\tau}(G / H) \simeq \mathrm{C}[Y]$. Rappelons maintenant que la conjecture (2.1) est vérifiée dans l'algèbre $\mathfrak{g}_{0}$ (voir [15]), ainsi

$$
D_{\tau}(G / H) \simeq \mathrm{C}\left[\Gamma_{\tau_{0}}\right]^{H} \simeq \mathrm{C}\left[P_{Y}\right],
$$

où $P_{Y}$ est la fonction polynomiale donnée par: $P_{Y}(\phi)=\phi(Y)$.

Soient $\left\{X_{1}^{\star}, \ldots, X_{p}^{\star}\right\}$ la base duale à $\mathcal{B}=\left\{X_{1}, \ldots, X_{p}\right\}$ et $\phi=f+\left(\phi_{1}, \ldots\right.$, $\left.\phi_{p-1}, \phi_{p}\right)=f+\phi_{1} X_{1}^{\star}+\cdots+\phi_{p-1} X_{p-1}^{\star}+\phi_{p} X_{p}^{\star} \in \Gamma_{\tau}$. Comme on peut supposer que $f$ est nulle sur vect $\left(X_{1}, \ldots, X_{p}\right)$, on peut écrire que

$$
\phi=\phi_{0}+\phi_{p} X_{p}^{\star}=\left(\phi_{0}, \phi_{p}\right) .
$$

Si les multiplicités qui apparaissent dans la désintégration de $\tau$ sont infinies, les $H$-orbites génériques de $\Gamma_{\tau}$ sont non-saturées par rapport à $\mathfrak{g}_{0}$. Ainsi, il existe un polynôme $H$-invariant non-nul sur les éléments génériques de $\Gamma_{\tau}$ qu'on peut écrire sous la forme

$$
P(\phi)=P\left(\phi_{0}, \phi_{p}\right)=P_{0}\left(\phi_{0}\right) \phi_{p}+Q_{0}\left(\phi_{0}\right)
$$

pour certaines fonctions polynomiales $P_{0}$ et $Q_{0}$ dans la variable $\phi_{0}$ qu'on regarde dans $\Gamma_{\tau_{0}}$. Comme la restriction de $P \operatorname{sur} \Gamma_{\tau_{0}}$ doit être toujours $H$-invariante et compte tenu de ce qui précède, on peut écrire que

$$
P_{0}=\sum_{\alpha} C_{\alpha} P_{Y}^{\alpha}, Q_{0}=\sum_{\beta} C_{\beta}^{\prime} P_{Y}^{\beta}
$$

où $C_{\alpha}$ et $C_{\beta}^{\prime}$ sont des coefficients complexes. Il s'ensuit que le polynôme $P^{\prime}(\phi)=\phi_{p}$ est $H$-invariant et donc $[X, \mathfrak{h}] \subset \mathfrak{h} \cap \operatorname{ker} f$, et ceci est alors absurde.

Supposons enfin que $\mathfrak{h} \not \subset \mathfrak{g}_{0}$. Dans ce cas $Y \notin \mathfrak{h}$, on pose $\mathfrak{h}^{\prime}=\left(\mathfrak{h} \cap \mathfrak{g}_{0}\right) \oplus \mathbf{R} Y$ et $H^{\prime}=\exp \mathfrak{h}^{\prime}$. On sait que les induites $\operatorname{Ind}_{H}^{G} \chi_{f}$ et $\tau^{\prime}=\operatorname{Ind}_{H^{\prime}}^{G} \chi_{f}$ sont équivalentes. L'opérateur $T_{H^{\prime}, H}$ qui entrelace ces deux induites est défini pour tout $\xi \in C_{c}^{\infty}(G / H, f)=$ $K(f, \mathfrak{h}, G) \cap C^{\infty}(G)$ et $g \in G$ par

$$
T_{H^{\prime}, H} \xi(g)=\int_{H^{\prime} / H^{\prime} \cap H} \xi(g h) \chi_{f}(h) \mathrm{d} \dot{h}=\int_{\mathbf{R}} \xi(g \exp (t Y)) \mathrm{d} t .
$$

Cet opérateur est une isométrie, d'après un choix propice des mesures sur $G / H$ et $G / H^{\prime}$. Les algèbres $D_{\tau}(G / H)$ et $D_{\tau^{\prime}}\left(G / H^{\prime}\right)$ sont par conséquent isomorphes, en effet l'application

$$
T_{H, H^{\prime}}^{\star}: D_{\tau^{\prime}}\left(G / H^{\prime}\right) \longrightarrow D_{\tau}(G / H)
$$

définie pour tout $D \in D_{\tau^{\prime}}\left(G / H^{\prime}\right)$ par:

$$
T_{H, H^{\prime}}^{\star} D=\left(T_{H^{\prime}, H}\right)^{-1} \circ D \circ T_{H^{\prime}, H}
$$

est un isomorphisme d'algèbre, donc $D_{\tau^{\prime}}\left(G / H^{\prime}\right)$ est abélienne et $\tau^{\prime}$ est à multiplicités finies d'après l'hypothèse de récurrence. Ainsi $\tau$ est encore à multiplicités finies. 
Nous allons regarder maintenant l'assertion (2.4) et la prouver dans le cas particulier où $\operatorname{dim} \mathfrak{h} \leqslant 2$. Il est bien clair que la preuve de cette assertion nous permet de donner une réponse positive à la question 2.2.

THÉORÈME 4.13. Supposons que g est nilpotente. Alors l'assertion (2.4) est vraie lorsque $\mathfrak{h}$ est une sous-algèbre telle que dim $\mathfrak{h} \leqslant 2$.

Preuve. Gardons les notations introduites au début de cette section et commençons tout d'abord par le cas où $\operatorname{dim} \mathfrak{h}=1$. Soit $j_{0}$ le premier indice pour lequel $\left[\mathfrak{h}, X_{j_{0}}\right] \neq\{0\}$. Si un tel $j_{0}$ n'existe pas, $\mathfrak{h}$ est central dans $\mathfrak{g}$ et rien à faire de plus. Alors, pour $j<j_{0},\left[\mathfrak{h}, X_{j}\right]=\{0\}$ et $X_{j} \in \mathcal{U}(\mathfrak{g}, \tau)$. Si notre algèbre s'agrandit au passage de $\mathfrak{f}_{j_{0}-1}$ à $\mathfrak{f}_{j_{0}}$, il existerait d'après ce qu'on a vu précédemment un élément $W \in \mathcal{U}(\mathfrak{g}, \tau) \cap \mathcal{U}\left(\mathfrak{f}_{j_{0}}\right)$ de la forme (5) avec $j=j_{0}$. Comme $U, V \in \mathcal{U}(\mathfrak{g}, \tau)$, on constate que $X_{j_{0}} \in \mathcal{U}(\mathrm{g}, \tau)$ car $\mathcal{U}(\mathrm{g}, \tau)$ ne possède aucun diviseur de zéro non-trivial. Mais cette dernière appartenance entraîne que $\left[\mathfrak{h}, X_{j_{0}}\right]=\{0\}$ ce qui est absurde. Pour $j>j_{0}$, les $H$-orbites génériques dans $\Gamma_{j}$ sont non-saturées par rapport à $\mathfrak{j}_{j-1}$ et, puisque le couple $(\mathfrak{g}, \mathfrak{h})$ est réductif, on peut facilement construire un élément $W \in \mathcal{U}(\mathfrak{g}, \tau) \cap \mathcal{U}\left(\mathfrak{f}_{j}\right)$ ayant la forme (5) à l'aide de la symétrisation.

Regardons maintenant le cas où $\operatorname{dim} \mathfrak{h}=2$ et proposons-nous de montrer la propriété dans ce cas-ci. Si $\mathfrak{h}_{1}$ est central dans $\mathfrak{g}$, nous sommes essentiellement ramené au cas où $\operatorname{dim} \mathfrak{h}=1$ et le résultat s'ensuit. Tout comme avant, soit $j_{0}$ le premier indice pour lequel $\left[\mathfrak{h}_{1}, X_{j_{0}}\right] \neq\{0\}$. S'il existe $q<j_{0}$ tel que $\left[\mathfrak{h}, X_{q}\right] \not \subset \mathfrak{h}_{1} \cap \operatorname{ker} f$ et que $X_{j} \in \mathcal{U}(\mathfrak{g}, \tau)$ pour $j<q$, il est évident que notre algèbre ne s'agrandit pas au passage de $\mathfrak{f}_{q-1}$ à $\mathfrak{f}_{q}$. Dans ce cas là, notons bien que les $H$-orbites génériques dans $\Gamma_{q}$ sont saturées par rapport à $\mathfrak{f}_{q-1}$.

Soit $q<j<j_{0}$. Remarquons d'abord que les $H$-orbites génériques dans $\Gamma_{j}$ sont non-saturées par rapport à $\mathfrak{f}_{j-1}$. Puisque $\left[\mathfrak{h}_{1}, X_{q}\right]=\{0\}$ et que $\left[\mathfrak{h}, X_{q}\right] \not \subset \mathfrak{h}_{1} \cap \operatorname{ker} f$, le critère du théorème (4.4) nous assure qu'il existe un élément $W \in \mathcal{U}(\mathfrak{g}, \tau) \cap \mathcal{U}\left(\mathfrak{f}_{j}\right)$ ayant la forme (5).

Comme $\left[\mathfrak{G}_{1}, \mathfrak{f}_{j_{0-1}}\right]=\{0\}$, les $H$-orbites génériques dans $\Gamma_{j_{0}}$ sont saturées par rapport à $\mathfrak{f}_{j_{0-1}}$ et il est clair que notre algèbre ne s'agrandit pas à l'étape de $\mathfrak{f}_{j_{0}}$ d'après l'argument fait pour le cas où $\operatorname{dim} \mathfrak{h}=1$ et appliqué à $\mathfrak{h}_{1}$.

Soit maintenant $j>j_{0}$. Les $H$-orbites génériques dans $\Gamma_{j}$ sont non-saturées par rapport à $\mathfrak{f}_{j-1}$ et conformément à ce fait, le critère du théorème (4.4) s'applique pour produire un nouvel élément $W \in \mathcal{U}(\mathfrak{g}, \tau) \cap \mathcal{U}\left(\mathfrak{f}_{j}\right)$ ayant la forme (5).

Avant de continuer nos observations, résumons brièvement ce qui se passe à l'étape $j=j_{0}$ :

(1) $\left[\mathfrak{h}_{1}, \mathfrak{j}_{j_{0}}\right] \neq\{0\}$ mais $\left[\mathfrak{h}_{1}, \mathfrak{f}_{j_{0}-1}\right]=\{0\}$.

(2) Les $H_{1}$-orbites génériques dans $\Gamma_{j_{0}}$ sont saturées par rapport à $\mathfrak{j}_{j_{0}-1}$.

(3) $D_{\tau_{j_{0}}}\left(K_{j_{0}} / H\right)=D_{\tau_{j_{0}-1}}\left(K_{j_{0}-1} / H\right)$.

Supposons maintenant que $\left[\mathfrak{h}, \mathfrak{f}_{j_{0}-1}\right] \subset \mathfrak{h}_{1} \cap \operatorname{ker} f$ et soit $r>j_{0}$ le premier indice pour lequel les $H$-orbites génériques dans $\Gamma_{r}$ sont saturées par rapport à $\mathfrak{f}_{r-1}$. S'il n'existe pas de tel indice, on se retrouve à chaque étape $\mathfrak{f}_{j}\left(j>j_{0}\right)$ dans la situation 
déjà étudiée plus haut. Allons constater que notre algèbre ne s'agrandit pas à l'étape $\mathfrak{f}_{r}$. Soit $j_{0}<j<r$. La situation au niveau $\mathfrak{f}_{j}$ étant celle que nous avons examiné plus haut, on a $\left[X_{j}, Y_{1}\right]=0$ ou $\left[X_{j_{0}}, Y_{2}\right] \in \mathfrak{h}_{1} \cap \operatorname{ker} f$, et en tout cas $\left[X_{j}, Y_{2}\right] \in \mathfrak{h}_{1} \cap \operatorname{ker} f$. Si $\left[X_{j_{0}}, Y_{2}\right] \in \mathfrak{h}_{1} \cap \operatorname{ker} f$, nous voyons aussitôt le résultat cherché car $\left[X_{r}, Y_{2}\right] \notin$ $\mathfrak{h}_{1} \cap \operatorname{ker} f$. Ensuite si $Y_{1}=\left[X_{j_{0}}, Y_{2}\right]$ et $f\left(Y_{1}\right) \neq 0$, alors $\left[X_{j}, Y_{2}\right]=0$ pour $j_{0}<j<r$ et $\left[X_{r}, Y_{1}\right] \in \mathfrak{f}_{j_{0}-1}$. Soit $X_{s}=\left[X_{j_{0}}, Y_{1}\right]$. Ecrivons

$$
\left[X_{r}, Y_{1}\right]=\sum_{k=1}^{j_{0}-1} \alpha_{k} X_{k}
$$

et

$$
\left[X_{r}, Y_{2}\right]=\sum_{k=1}^{r-1} \beta_{k} X_{k}+\gamma Y_{1}
$$

avec certains $\alpha_{k}, \beta_{k}, \gamma \in \mathbf{R}$. Comme

$$
\left[\left[X_{r}, Y_{1}\right], Y_{2}\right]=\left[\left[X_{r}, Y_{2}\right], Y_{1}\right] \text {, }
$$

on a que $\beta_{j_{0}}=0$. Supposons que $\left[X_{r}, Y_{1}\right] \neq 0$, sinon le résultat cherché s'obtient aussitôt.

Supposons pour le moment qu'il existe $W \in \mathcal{U}(\mathfrak{g}, \tau)$ ayant la forme $W=X_{r} U+V$ avec $U, V \in \mathcal{U}\left(\mathfrak{f}_{r-1}\right)$ vérifiant $U \notin \mathcal{U}(\mathrm{g}) \mathfrak{a}_{\tau}$. Il se réduit aux cas où $V=X_{j_{0}} \tilde{V}$ et où $U, \tilde{V}$ s'expriment par les éléments $X_{i}\left(1 \leqslant i \leqslant r-1, i \neq j_{0}\right)$. De $\left[W, Y_{1}\right] \in \mathcal{U}(\mathrm{g}) \mathfrak{a}_{\tau}$, il vient que

$$
\left(\sum_{k=1}^{j_{0}-1} \alpha_{k} X_{k}\right) U+X_{s} \tilde{V} \in \mathcal{U}(\mathfrak{g}) \mathfrak{a}_{\tau} .
$$

$\operatorname{De}\left[W, Y_{2}\right] \in \mathcal{U}(\mathfrak{g}) \mathfrak{a}_{\tau}$, on a que

$$
\left(\sum_{k=1}^{r-1} \beta_{k} X_{k}+\gamma Y_{1}\right) U-\sqrt{-1} f\left(Y_{1}\right) \tilde{V} \in \mathcal{U}(\mathfrak{g}) \mathfrak{a}_{\tau} .
$$

D'où

$$
\sqrt{-1} f\left(Y_{1}\right)\left(\sum_{k=1}^{j_{0}-1} \alpha_{k} X_{k}\right)+X_{s}\left(\sum_{k=1}^{r-1} \beta_{k} X_{k}+\gamma Y_{1}\right) \in \mathcal{U}(\mathfrak{g}) \mathfrak{a}_{\tau} .
$$

Cette appartenance est possible seulement si $\sum_{k=1}^{r-1} \beta_{k} X_{k}=0$, d'où l'on déduit facilement le résultat voulu en remplaçant $X_{r} \operatorname{par} X_{r}-\gamma X_{j_{0}}$.

Supposons désormais que $\left[X_{j_{0}}, Y_{1}\right]$ et $\left[X_{j_{0}}, Y_{2}\right]$ sont linéairement indépendants modulo $\mathfrak{h}_{1}$, soient $\left[X_{j_{0}}, Y_{1}\right]=X_{s}$ et $\left[X_{j_{0}}, Y_{2}\right]=X_{t}+c Y_{1}(c \in \mathbf{R}, s \neq t)$. Ecrivons encore une fois

$$
\left[X_{r}, Y_{1}\right]=\sum_{k=1}^{r-1} \alpha_{k} X_{k},\left[X_{r}, Y_{2}\right]=\sum_{k=1}^{r-1} \beta_{k} X_{k}+\gamma Y_{1}\left(\alpha_{k}, \beta_{k}, \gamma \in \mathbf{R}\right) .
$$


Comme $\left[\left[X_{r}, Y_{1}\right], Y_{2}\right]=\left[\left[X_{r}, Y_{2}\right], Y_{1}\right]$, nous avons que

$$
\alpha_{j_{0}}\left(X_{t}+c Y_{1}\right)+\delta Y_{1}=\beta_{j_{0}} X_{s},
$$

avec un certain $\delta \in \mathbf{R}$, d'où $\alpha_{j_{0}}=\beta_{j_{0}}=0$. Compte tenu de ce fait, si notre algèbre s'agrandit à l'étape de $\mathfrak{f}_{r}$, on $y$ trouverai un élément $W \in \mathcal{U}(\mathfrak{g}, \tau)$ ayant la forme $W=X_{r} U+X_{j_{0}} V$ avec $U, V \in \mathcal{U}\left(\mathfrak{f}_{r-1}\right)$ qui s'expriment par les éléments $X_{i},(1 \leqslant$ $\left.i \leqslant r-1, i \neq j_{0}\right)$ et vérifiant $U \notin \mathcal{U}(\mathfrak{g}) \mathfrak{a}_{\tau}$. De $\left[W, Y_{1}\right] \in \mathcal{U}(\mathfrak{g}) \mathfrak{a}_{\tau}$, il vient que

$$
\left(\sum_{k=1}^{r-1} \alpha_{k} X_{k}\right) U+X_{s} V \in \mathcal{U}(\mathfrak{g}) \mathfrak{a}_{\tau} .
$$

De $\left[W, Y_{2}\right] \in \mathcal{U}(\mathfrak{g}) \mathfrak{a}_{\tau}$, nous avons que

$$
\left(\sum_{k=1}^{r-1} \beta_{k} X_{k}+\gamma Y_{1}\right) U+\left(X_{t}+c Y_{1}\right) V \in \mathcal{U}(\mathfrak{g}) \mathfrak{a}_{\tau} .
$$

Maintenant, il faut noter que

$$
\left[X_{s}, X_{t}+c Y_{1}\right]=\left[\left[X_{j_{0}}, Y_{1}\right],\left[X_{j_{0}}, Y_{2}\right]\right]=\left[X_{j_{0}},\left[\left[Y_{1},\left[X_{j_{0}}, Y_{2}\right]\right]\right]+\left[Y_{1},\left[\left[X_{j_{0}}, Y_{2}\right], X_{j_{0}}\right]\right]=0 .\right.
$$

Toutes ces observations nous donnent

$$
\left(X_{t}+c Y_{1}\right)\left(\sum_{k=1}^{r-1} \alpha_{k} X_{k}\right)-X_{s}\left(\sum_{k=1}^{r-1} \beta_{k} X_{k}+\gamma Y_{1}\right) \in \mathcal{U}(\mathfrak{g}) \mathfrak{a}_{\tau} .
$$

Lorsque $f\left(Y_{1}\right) \neq 0$, cette condition est remplie seulement si

$$
c \sum_{k=1}^{r-1} \alpha_{k} X_{k}-\gamma X_{s}=0
$$

A savoir, $\left[c X_{r}-\gamma X_{j_{0}}, Y_{1}\right]=0$. A cause de la saturation des $H$-orbites par rapport à $\mathfrak{f}_{r-1}$, on en tire le résultat attendu sauf dans le cas où $c=\gamma=0$. Supposons donc que $f\left(Y_{1}\right)=0$, ou $c=\gamma=0$ sinon. Alors la condition décrite ci-dessus est remplie seulement si

$$
X_{t}\left(\sum_{k=1}^{r-1} \alpha_{k} X_{k}\right) \equiv X_{s}\left(\sum_{k=1}^{r-1} \beta_{k} X_{k}\right)
$$

à savoir que

$$
\left(\left[X_{j_{0}}, Y_{2}\right]-c Y_{1}\right)\left[X_{r}, Y_{1}\right] \equiv\left[X_{j_{0}}, Y_{1}\right]\left(\left[X_{r}, Y_{2}\right]-\gamma Y_{1}\right) .
$$

La commutativité entre des éléments apparus dans cette égalité nous permet de la regarder dans l'algèbre symétrique $S(\mathfrak{g}) / S(\mathfrak{g}) \overline{\mathfrak{a}_{\tau}}$. Evaluons deux membres au point $l \in \Gamma_{r}$ générique et nous avons

$$
l\left(\left[X_{j_{0}}, Y_{2}\right]\right) l\left(\left[X_{r}, Y_{1}\right]\right)=l\left(\left[X_{j_{0}}, Y_{1}\right]\right) l\left(\left[X_{r}, Y_{2}\right]\right) .
$$


Cela étant, en posant $Y=l\left(\left[X_{j_{0}}, Y_{1}\right]\right) Y_{2}-l\left(\left[X_{j_{0}}, Y_{2}\right]\right) Y_{1}$, on constate aisément que

$$
l\left(\left[X_{j_{0}}, Y\right]\right)=l\left(\left[X_{r}, Y\right]\right)=0 .
$$

Mais cela contredit notre hypothèse que l'orbite $H \cdot l$ est saturée par rapport à $\mathfrak{f}_{r-1}$.

Soit enfin $r<j \leqslant p$. Ayant une fois passé l'étape $\mathfrak{f}_{r}$, on voit que les $H$-orbites génériques dans $\Gamma_{j}$ sont non saturées par rapport à $\mathfrak{f}_{j-1}$. Rappelons qu'on vient de vérifier la non-existence d'un élément $W \in \mathcal{U}(\mathfrak{g}, \tau)$ ayant la forme $W=X_{r} U+V$ avec $U, V \in \mathcal{U}\left(\mathfrak{f}_{r-1}\right)$ vérifiant $U \notin \mathcal{U}(\mathfrak{g}) \mathfrak{a}_{\tau}$. D'autre part, il existe $W \in \mathcal{U}\left(\mathfrak{f}_{r}\right)$ de cette même forme qui est $H_{1}$-invariant. Tout cela nous permet d'appliquer le critère du théorème (4.4) au niveau $\mathfrak{f}_{j}$ pour affirmer que notre algèbre s'agrandit à ce passage.

En résumé, gardons les notations et posons $K_{j}=\exp \left(\mathfrak{f}_{j}\right)$ et $\sigma_{j}=\operatorname{Ind}_{H}^{K_{j}} \chi_{f}$ pour $0 \leqslant j \leqslant p$. En particulier, $K_{0}=H, K_{p}=G$ et $\sigma_{0}=\chi_{f}, \sigma_{p}=\tau$. La suite d'inclusions des sous-groupes $H \subset K_{1} \subset \cdots \subset K_{p-1} \subset K_{p}=G$ entraîne celle des algèbres

$$
D_{\sigma_{1}}\left(K_{1} / H\right) \subset D_{\sigma_{2}}\left(K_{2} / H\right) \subset \cdots \subset D_{\sigma_{p-1}}\left(K_{p-1} / H\right) \subset D_{\sigma_{p}}\left(K_{p} / H\right)=D_{\tau}(G / H) .
$$

PROPOSITION 4.14. Supposons que g est une algèbre de Lie nilpotente et que h est une sous-algèbre telle que $\operatorname{dim} \mathfrak{h}=2$. Pour $1 \leqslant j \leqslant p, D_{\sigma_{j}}\left(K_{j} / H\right)=D_{\sigma_{j-1}}\left(K_{j-1} / H\right)$ si et seulement si les $H$-orbites génériques dans $\Gamma_{j}$ sont non-saturées par rapport à $\mathfrak{f}_{j-1}$.

COROLLAIRE 4.15. Supposons que g est une algèbre de Lie nilpotente et que $\mathfrak{h}$ est une sous-algèbre telle que dim $\mathfrak{h}=3$. Supposons que $\tau=\sigma_{p}$ est à multiplicités infinies tandis que $\sigma_{p-1}$ est à multiplicités finies. Si l'ensemble $\left\{l \in \Gamma_{\tau}: \mathfrak{h} \not \subset \mathfrak{h}_{2}+\mathfrak{g}(l)\right\}$ n'est pas négligeable pour la mesure de Lebesgue sur $\Gamma_{\tau}$, alors $D_{\sigma_{p-1}}\left(K_{p-1} / H\right) \neq D_{\tau}(G / H)$ et l'algèbre $D_{\tau}(G / H)$ n'est pas commutative.

Preuve. Soit $l \in \Gamma_{\tau}$ générique, et reprenons la notation $\mathfrak{g}^{\prime}$ au lieu de $\mathfrak{f}_{p-1}$. L'hypothèse sur les multiplicités implique qu'il existe $X \in \mathfrak{g} \backslash \mathfrak{g}^{\prime}$ vérifiant $l([X, \mathfrak{h}])=\{0\}$. S'il en est ainsi, $\mathfrak{h} \subset \mathfrak{h}_{2}+\mathfrak{g}^{\prime}\left(l_{\mid \mathfrak{g}^{\prime}}\right)$ entraîne $\mathfrak{h} \subset \mathfrak{h}_{2}+\mathfrak{g}(l)$. Donc sous nos hypothèses, l'ensemble des $l \in \Gamma_{p-1}$ où $\mathfrak{h}_{2}+\mathfrak{g}^{\prime}\left(l_{\mid \mathfrak{g}^{\prime}}\right)$ n'est pas un sous-espace lagrangien n'est pas négligeable. Cela signifie que $\tau_{2}=\operatorname{Ind}_{H_{2}}^{G^{\prime}} \chi_{f}$ est à multiplicités infinies. Soit $q(1 \leqslant q \leqslant p-1)$, le premier indice tel que $\operatorname{Ind}_{H_{2}}^{K_{q}} \chi_{f}$ soit à multiplicités infinies.

Nous en déduisons que, pour presque toutes les $l \in \Gamma_{q}$, l'orbite $K_{q} \cdot l$ (resp. $H \cdot l$ ) est saturée (resp. non-saturée) par rapport à $\mathfrak{f}_{q-1}$. Comme $\sigma_{p-1}$ est à multiplicités finies, le résultat dû à Corwin-Greenleaf dit que l'algèbre $D_{\sigma_{p-1}}\left(G^{\prime} / H\right)$ ne change pas au passage de $K_{q-1}$ à $K_{q}$. Pourtant la proposition (4.14) nous avertit que l'algèbre $D_{\tau_{2}}\left(G^{\prime} / H\right)$ s'agrandit à ce passage. D'où le théorème (4.4) nous apporte le résultat cherché.

Remarque 4.16. Concernant la deuxième condition $\mathcal{U}\left(\mathrm{g}^{\prime}, \tau_{d-1}\right) \not \subset \mathcal{U}\left(\mathrm{g}^{\prime}, \tau_{d}\right)$ du théorème (4.4), elle tombe en défaut lorsque $\tau_{d-1}$ est à multiplicités finies. Car dans ce cas $\mathcal{U}\left(\mathfrak{g}^{\prime}, \tau_{d-1}\right) / \mathcal{U}\left(\mathfrak{g}^{\prime}\right) \mathfrak{a}_{\tau_{d-1}}$ est commutative et $\mathfrak{h} \subset \mathcal{U}\left(\mathfrak{g}^{\prime}, \tau_{d-1}\right)$. 
Supposons que $\tau_{d-1}$ est à multiplicités finies et que

$$
\mathcal{U}(\mathfrak{g}, \tau) \not \subset \mathcal{U}\left(\mathfrak{g}^{\prime}\right)+\mathcal{U}(\mathfrak{g}) \mathfrak{a}_{\tau}
$$

Soit

$$
W^{\prime}=X_{p} U^{\prime}+V^{\prime} \in \mathcal{U}(\mathfrak{g})
$$

avec $U^{\prime}, V^{\prime} \in \mathcal{U}\left(\mathfrak{g}^{\prime}\right)$ vérifiant $U^{\prime} \notin \mathcal{U}(\mathfrak{g}) a_{\tau^{\prime}}$. Si $W^{\prime}$ satisfait aux conditions $\left[\mathfrak{h}_{d-1}, W^{\prime}\right] \subset \mathcal{U}(\mathfrak{g}) \mathfrak{a}_{\tau^{\prime}}$, alors on a nécessairement que $W^{\prime} \in \mathcal{U}(\mathfrak{g}, \tau)$. En effet, puisque

$$
\mathcal{U}(\mathfrak{g}, \tau) \not \subset \mathcal{U}\left(\mathfrak{g}^{\prime}\right)+\mathcal{U}(\mathfrak{g}) \mathfrak{a}_{\tau}
$$

il existe $W=X_{p} U+V \in \mathcal{U}(\mathfrak{g}, \tau)$ avec $U, V \in \mathcal{U}\left(\mathfrak{g}^{\prime}\right)$ vérifiant $U \notin \mathcal{U}(\mathfrak{g}) \mathfrak{a}_{\tau}$. D'où quitte à supposer que $U, U^{\prime} \in \mathcal{U}\left(\mathfrak{g}^{\prime}, \tau_{d-1}\right) \subset \mathcal{U}\left(\mathfrak{g}^{\prime}, \tau_{d}\right)$, la commutativité de $\mathcal{U}\left(\mathfrak{g}^{\prime}, \tau_{d-1}\right) /$ $\mathcal{U}\left(\mathfrak{g}^{\prime}\right) \mathfrak{a}_{\tau_{d-1}}$ nous assure que

$$
\tilde{V}=W^{\prime} U-W U^{\prime} \equiv V^{\prime} U-V U^{\prime}\left(\bmod \mathcal{U}(\mathfrak{g}) \mathfrak{a}_{\tau}\right)
$$

appartient à $\mathcal{U}\left(\mathfrak{g}^{\prime}, \tau_{d-1}\right) \subset \mathcal{U}\left(\mathfrak{g}^{\prime}, \tau_{d}\right)$. Ainsi,

$$
W^{\prime} U \equiv W U^{\prime}+\tilde{V}\left(\bmod \mathcal{U}(\mathfrak{g}) \mathfrak{a}_{\tau}\right)
$$

appartient à $\mathcal{U}(\mathfrak{g}, \tau)$ et par conséquent $\left[Y, W^{\prime}\right] U \in \mathcal{U}(\mathfrak{g}) \mathfrak{a}_{\tau}$ pour tout $Y \in \mathfrak{h}$. Comme $\mathcal{U}(\mathfrak{g}, \tau) / \mathcal{U}(\mathfrak{g}) \mathfrak{a}_{\tau}$ n'a pas de diviseurs de zéro non-trivial, il s'ensuit que $W^{\prime} \in \mathcal{U}(\mathfrak{g}, \tau)$. En effet, il vient que

$$
\left[Y, W^{\prime}\right]=\left[Y, X_{p}\right] U^{\prime}+X_{p}\left[Y, U^{\prime}\right]+\left[Y, V^{\prime}\right] \in \mathcal{U}\left(\mathfrak{g}^{\prime}\right)+\mathcal{U}(\mathfrak{g}) \mathfrak{a}_{\tau} .
$$

Ensuite, pour $Y^{\prime} \in \mathfrak{h}^{\prime}=\mathfrak{h}_{d-1}$,

$$
\left[Y^{\prime},\left[Y, W^{\prime}\right]\right]=\left[\left[Y^{\prime}, Y\right], W^{\prime}\right]+\left[Y,\left[Y^{\prime}, W^{\prime}\right]\right] \in \mathcal{U}(\mathfrak{g}) \mathfrak{a}_{\tau^{\prime}} .
$$

Tout compte fait,

$$
\left[Y, W^{\prime}\right] \in \mathcal{U}\left(\mathfrak{g}^{\prime}, \tau_{d-1}\right)+\mathcal{U}(\mathfrak{g}) \mathfrak{a}_{\tau} \subset \mathcal{U}\left(\mathfrak{g}^{\prime}, \tau_{d}\right)+\mathcal{U}(\mathfrak{g}) \mathfrak{a}_{\tau} \subset \mathcal{U}(\mathfrak{g}, \tau)
$$

comme $\tau_{d-1}$ est à multiplicités finies (voir le début de la remarque).

Cela posé, supposons que $\tau$ (resp. $\tau_{d}, \tau_{d-1}$ ) est à multiplicités infinies (resp. finies). Si on trouve un $W^{\prime}=X_{p} U^{\prime}+V^{\prime} \in \mathcal{U}\left(\mathfrak{g}, \tau^{\prime}\right)$ avec $U^{\prime}, V^{\prime} \in \mathcal{U}\left(\mathfrak{g}^{\prime}\right)$ vérifiant $U^{\prime} \notin \mathcal{U}(\mathfrak{g}) \mathfrak{a}_{\tau^{\prime}}$ et tel que $\left[Y_{d}, W^{\prime}\right] \notin \mathcal{U}(\mathfrak{g}) \mathfrak{a}_{\tau}$, alors la conjecture de Duflo-Corwin-Greenleaf ne s'établirait pas.

\section{Sur l'algèbre $D_{\pi}(G)^{H}$}

Soit $\pi$ une représentation unitaire et irréductible d'un groupe de Lie résoluble exponentiel $G=\exp g$ associée à une $G$-orbite coadjointe $\Omega(\pi) \subset \mathfrak{g}^{\star}$ d'une forme linéaire $f$. Soit $\operatorname{ker}(\mathrm{d} \pi)$ l'idéal primitif bilatère de l'algèbre enveloppante $\mathcal{U}(\mathfrak{g})$. Soit encore $H$ un sous-groupe analytique de $G$ d'algèbre de Lie $\mathfrak{h}$. Tout comme dans le paragraphe 2, on définit $D_{\pi}(G)^{H}$ l'algèbre étant l'algèbre image par l'homomorphisme $\mathrm{d} \pi$ du quotient $\mathcal{U}_{\pi}(\mathrm{g})^{\mathfrak{h}} / \operatorname{ker}(\mathrm{d} \pi)$. Ici,

$$
\mathcal{U}_{\pi}(\mathfrak{g})^{\mathfrak{h}}=\{A \in \mathcal{U}(\mathfrak{g}):[A, \mathfrak{h}] \subset \operatorname{ker}(\mathrm{d} \pi)\}
$$


Nous allons donner une description explicite de l'algèbre $D_{\pi}(G)^{H}$ quand $G$ est un groupe de Lie nilpotent. Soit

$$
\{0\}=\mathfrak{g}_{0} \subset \mathfrak{g}_{1} \subset \cdots \subset \mathfrak{g}_{m-1} \subset \mathfrak{g}_{m}=\mathfrak{g}
$$

une suite de Jordan-Hölder de $\mathfrak{g}$, c.à.d une suite décroissante d'idéaux de $\mathfrak{g}$ telle que $\operatorname{dim}\left(\mathfrak{g}_{j}\right)=j, j=0, \ldots, m$. Soit $f \in \mathfrak{g}^{\star}$, on définit $e(f)=\left\{1 \leqslant j \leqslant m \mid \mathfrak{g}_{j} \neq \mathfrak{g}_{j-1}+\mathfrak{g}(f)\right\}$.

Soit $X_{j} \in \mathfrak{g}_{j} \backslash \mathfrak{g}_{j-1}, j=1, \ldots, m$. Alors $\left\{X_{1}, \ldots, X_{m}\right\}$ est une base de Jordan-Hölder de $g$ et on a que

$$
j \in e(f) \Leftrightarrow X_{j} \notin \mathfrak{g}_{j-1}+\mathfrak{g}(f) .
$$

Posons $\Omega_{e}=\left\{l \in \mathfrak{g}^{\star}: e(l)=e\right\}$. Si $f \in \mathfrak{g}^{\star}$ avec $e(f) \neq \emptyset$ i.e $\mathfrak{g}(f) \neq \mathfrak{g}$ et si $e=$ $e(f)=\left\{j_{1}<\cdots<j_{d}\right\}$, alors $\left\{X_{j_{1}}, \ldots, X_{j_{d}}\right\}$ est une base de $\mathfrak{g}(\bmod \mathfrak{g}(f))$. Il existe des fonctions $R_{j}^{e}: \Omega_{e} \times \mathbf{R}^{d} \rightarrow \mathbf{R}, j=1, \ldots, m$ telles que:

a) Pour $f \in \Omega_{e}$ fixée, $\left(x_{1}, \ldots, x_{d}\right) \mapsto R_{j}^{e}\left(f, x: \mathbf{R}^{d} \rightarrow \mathbf{R}\right.$ est une fonction polynomiale en $x=\left(x_{1}, \ldots, x_{d}\right)$ et les coefficients sont des fonctions $G$-invariantes sur $\Omega_{e}, i \in\{1, \ldots, m\}$

b) $R_{j}^{e}(f, x)=x_{k}$ pour $j=j_{k} \in e, f \in \Omega_{e}$;

c) Si $1 \leqslant i \leqslant m, j_{k} \leqslant j<j_{k+1}$ alors $R_{j}^{e}(f, x)$ ne dépend que de $x_{1}, \ldots, x_{k}$;

d) Pour tout $f \in \Omega_{e}$, l'orbite coadjointe $G \cdot f$ est donnée par:

$$
G \cdot f=\left\{\sum_{i=1}^{m} R_{j}^{e}(f, x) l_{i} \mid x \in \mathbf{R}^{d}\right\},
$$

où $\left\{l_{1}, \ldots, l_{m}\right\}$ est une base de $\mathrm{g}^{\star}$ telle que $l_{i}\left(X_{j}\right)=\delta_{i, j,} 1 \leqslant i, j \leqslant m$. (voir [24])

Soit $r_{j}^{e}(f)$ l'image dans $\mathcal{U}(\mathrm{g})$ par la symétrisation de l'élément $R_{j}^{e}\left(f,-i X_{j_{1}}, \ldots\right.$, $\left.-i X_{j_{d}}\right)$ dans l'algèbre symétrique $\mathcal{S}(\mathfrak{g})$ de $\mathfrak{g}_{\mathrm{C}}$, c'est à dire on remplace la variable $x_{k}$ dans $R_{j}^{e}(f, x)$ par $-i X_{j_{k}}$. Notons en particulier que $r_{j_{k}}^{e}(f)=-i X_{j_{k}}$. Soit $G_{e}$ le sous-espace de $S\left(\mathfrak{g}_{\mathrm{C}}\right)$ engendré par les éléments de la forme $X_{j_{1}}^{\alpha_{1}} \cdots X_{j_{d}}^{\alpha_{d}}$, $\left(\alpha_{1}, \ldots, \alpha_{d}\right) \in \mathrm{IN}^{d}$, et soit $F_{e}$ l'image dans $\mathcal{U}(\mathrm{g})$ de $G_{e}$ par la symétrisation. D'autre part, soit $E_{e}$ le sous-espace de $\mathcal{U}(\mathfrak{g})$ engendré par les éléments de la forme $X_{j_{1}}^{\alpha_{1}} \cdots X_{j_{d}}^{\alpha_{d}}, \alpha_{1}, \ldots, \alpha_{d} \in \mathrm{IN}^{d}$. Si $e=\emptyset$, on pose $G_{e}=F_{e}=E_{e}=\mathrm{C} \cdot 1$.

Pedersen a prouvé que l'idéal primitif $\operatorname{ker}(\mathrm{d} \pi)$, où $\pi \in \hat{G}$ telle que $f \in \Omega_{G}(\pi)$, est engendré par les éléments $u_{j}^{e}(f)=X_{j}-\operatorname{ir}_{j}^{e}(f), j \notin e$ et que

$$
\mathcal{U}(\mathrm{g})=\operatorname{ker}(\mathrm{d} \pi) \oplus E_{e}=\operatorname{ker}(\mathrm{d} \pi) \oplus F_{e},
$$

(voir [24, Theorem 2.1.1 et Theorem 2.2.1]). De même, l'action de d $\pi$ sur $E_{e}$ et $F_{e}$ est fidèle (voir [24, Lemma 2.2.12 et Lemma 2.2.13]). Ainsi, nous obtenons le lemme suivant:

LEMME 5.1. Soit $G=\exp (\mathfrak{g})$ un groupe de Lie nilpotent connexe et simplement connexe. Soit $H=\exp (\mathfrak{h})$ un sous-groupe analytique de $G$ et $\pi$ une représentation unitaire et irréductible de G. Posons $e=e(f)$ avec $f \in \Omega(\pi)$. Alors

$$
\begin{gathered}
D_{\pi}(G)^{H} \simeq E_{e}^{H} \simeq F_{e}^{H}, \\
\text { où } E_{e}^{H}=E_{e} \cap \mathcal{U}_{\pi}(\mathfrak{g})^{\mathfrak{h}} \text { et } F_{e}^{H}=F_{e} \cap \mathcal{U}_{\pi}(\mathfrak{g})^{\mathfrak{h}} .
\end{gathered}
$$


Comme il a été mentionné dans le deuxième paragraphe, nous voudrons comprendre la structure de l'algèbre $D_{\pi}(G)^{H}$ en terme d'orbites coadjointes pour le spectre et les multiplicités de $\pi_{\mid H}$. Plus précisement, nous allons discuter en ce lieu la question suivante:

Quand est-ce que l'algèbre $D_{\pi}(G)^{H}$ est commutative?

Nous allons répondre à cette question lorsque $H$ est un sous-groupe normal de $G$. En fait, nous croyons que l'assertion suivante doit être vraie:

CONJECTURE 5.2. Soit $G=\exp (\mathfrak{g})$ un groupe de Lie nilpotent connexe et simplement connexe. Soit $H=\exp (\mathfrak{h})$ un sous-groupe analytique de $G$ et $\pi$ une représentation unitaire et irréductible de G. Alors, l'algèbre $D_{\pi}(G)^{H}$ est commutative si et seulement si les multiplicités qui apparaissent dans la désintégration de $\pi_{\mid H}$ sont finies $\eta$-presque partout.

En passant, remarquons le phénomène suivant:

LEMME 5.3. Soit $G=\exp (\mathfrak{g})$ un groupe de Lie résoluble exponentiel d'algèbre de Lie g. Soit $H=\exp (\mathfrak{h})$ un sous-groupe analytique de $G$ et $\pi$ une représentation unitaire et irréductible de G. Si $\pi_{\mid H}$ est irréductible, alors l'algèbre $D_{\pi}(G)^{H}$ est triviale.

Preuve. Soit $\mathrm{d} \pi(A) \in D_{\pi}(G)^{H}$, alors utilisant le fait que $A \in \mathcal{U}_{\pi}(\mathfrak{g}, \mathfrak{h})$, on peut déduire que $\mathrm{d} \pi(A)$ commute avec $\mathrm{d} \pi(X)$ pour tout $X \in \mathfrak{h}$. Par le lemme de Schur, on obtient $\mathrm{d} \pi(A) \in \mathrm{C} \cdot 1$ et finalement $\mathcal{U}_{\pi}(\mathfrak{g}, \mathfrak{h})=\mathrm{C} \cdot 1+\operatorname{ker} \mathrm{d} \pi$, d'où le résultat.

PREUVE DU THÉORÈME 5.4 (2.5). Nous allons utiliser une récurrence sur la dimension de $\mathfrak{g}$. Notons $\mathfrak{a}(f)$ le plus grand idéal de $\mathfrak{g}$ contenu dans $\mathfrak{g}(f)$, manifestement,

$$
\mathfrak{a}(f)=\bigcap_{g \in G} A d(g)(\mathfrak{g}(f))=\bigcap_{\phi \in \Omega(\pi)} \mathfrak{g}(\phi) .
$$

Soit encore $\mathfrak{a}=\mathfrak{a}(f) \cap \mathfrak{h}$ qui est un idéal de $\mathfrak{g}$. Si $\mathfrak{a}=\mathfrak{h}$ alors $\mathfrak{h} \subset \mathfrak{a}(f)$ et tout point dans $\Omega_{G}(\pi) \cap p^{-1}\left(\Omega_{H}(\sigma)\right)$ est une $H$-orbite. Cela veut dire que les multiplicités de $\pi_{\mid H}$ sont infinies, ce qui est impossible compte tenu de notre hypothèse. Ainsi, nous pouvons supposer que $\mathfrak{a} \neq \mathfrak{h}$.

Soit $\left\{Z_{1}, \ldots, Z_{n}\right\}$ une base de Jordan-Hölder de $\mathfrak{g}$ passant à travers $\mathfrak{a}$ et $\mathfrak{h}$ de telle manière que $\mathfrak{a}=\operatorname{span}\left(Z_{k+1}, \ldots, Z_{n}\right)$ et $\mathfrak{h}=\operatorname{span}\left(Z_{\left.p, \ldots, Z_{n}\right)}(p \leqslant k)\right.$. Soit $Y=$ $Z_{k} \in \mathfrak{h} \backslash \mathfrak{a}$ et

$$
\mathfrak{g}^{Y}=\{T \in \mathfrak{g}: f([T, Y])=0\} .
$$

En fait, $\mathfrak{g}^{Y} \neq \mathfrak{g}$. Sinon, nous avons $f([\mathfrak{g}, Y])=0$ et alors $Y \in \mathfrak{g}(f)$ et finalement $\mathbf{R} Y \oplus \mathfrak{a}$ est un idéal contenu dans $\mathfrak{a}$, ce qui est impossible. Il s'ensuit que $\mathfrak{g}^{Y}$ est un idéal de codimension 1 dans g. 
LEMME 5.5. Avec les mêmes notations, nous avons

$$
\mathcal{U}_{\pi}(\mathfrak{g})^{\mathfrak{h}} \subset \mathcal{U}\left(\mathfrak{g}^{Y}\right) .
$$

Preuve. Nous choisissons $X \in \mathfrak{g} \backslash \mathfrak{g}^{Y}$ tel que $f([X, Y])=1$. Soit $W \in \mathcal{U}_{\pi}(\mathfrak{g})^{\mathfrak{h}}$ de la forme $W=\sum_{k=0}^{m} X^{k} V_{k}$ avec certains $V_{k} \in \mathcal{U}\left(\mathfrak{g}^{Y}\right)$. Calculons [ $\left.W, Y\right]$ qui appartient à $\operatorname{ker}(\mathrm{d} \pi)$. Tout d'abord, par un simple calcul, on voit que $\left[X^{m}, Y\right]=m X^{m-1}$ $[X, Y]+Q$ où $Q \in \mathcal{U}(\mathfrak{g})$ de degré inférieur ou égal à $m-2$ en $X$. Il s'ensuit qu'il existe $A \in \mathcal{U}(\mathfrak{g})$ de degré inférieur ou égal à $m-2$ en $X$ tel que

$$
[W, Y]=m X^{m-1} V_{m}[X, Y]+A .
$$

Puisque $[X, Y] \in \mathfrak{a}(f)$ et $f([X, Y])=1$, nous pouvons conclure que $V_{m} \in \operatorname{ker}(\mathrm{d} \pi)$ et inductivement que $W \in \mathcal{U}\left(\mathfrak{g}^{Y}\right)$ modulo $\operatorname{ker}(\mathrm{d} \pi)$. Ici bien évidemment nous avons utilisé le fait que $\left[\mathcal{U}\left(\mathfrak{g}^{Y}\right), Y\right] \subset \operatorname{ker}(\mathrm{d} \pi)$ qui n'est pas difficile à vérifier.

Notons que nous avons $\pi \simeq \operatorname{Ind}_{G^{Y}}^{G} \pi_{1}$, où $G^{Y}=\exp \left(g^{Y}\right)$ et $\pi_{1} \in \hat{G}^{Y}$ associée à $f_{1}=f_{\mid \mathfrak{g}^{Y}}$ par la théorie de Kirillov.

Choisissons un supplémentaire $\mathrm{t}$ de $\mathfrak{g}^{Y}$ dans $\mathfrak{g}$ tel que $T G^{Y}=G$ où $T=\exp (\mathrm{t})$. Maintenant nous allons étudier les deux cas possibles qui peuvent avoir lieu.

Cas 1: $\mathfrak{h} \subset \mathfrak{g}^{Y}$.

Ecrivons

$$
\pi_{\mid H}=\left(\pi_{\mid G^{Y}}\right)_{\mid H} \simeq \int_{\mathbf{R}}^{\oplus} \pi_{t \mid H} \mathrm{~d} t .
$$

Ici $\pi_{t}$ est la représentation associée à $\operatorname{Ad}^{\star}(\exp (t X)) f_{\mid \mathfrak{g}^{Y}}$ dans le groupe $G^{Y}$. Par le fait que les multiplicités de $\pi_{\mid H}$ sont finies, on peut déduire que les multiplicités de $\pi_{t \mid H}$ sont finies pour presque tout $t \in \mathbf{R}$. Montrons que

$$
\mathcal{U}_{\pi}(\mathfrak{g})^{\mathfrak{h}}=\bigcap_{t \in \mathbf{R}} \mathcal{U}_{\pi_{t}}\left(\mathfrak{g}^{Y}\right)^{\mathfrak{h}}
$$

Tout d'abord, rappelons que pour tout $A \in \mathcal{U}\left(\mathrm{g}^{Y}\right)$ nous avons que:

$$
\mathrm{d} \pi(A)=\int_{\mathbf{R}}^{\oplus} \mathrm{d} \pi_{t}(A) \mathrm{d} t .
$$

Pour $A \in \mathcal{U}_{\pi}(\mathfrak{g})^{\mathfrak{h}}$, nous avons

$$
0=\mathrm{d} \pi([A, \mathfrak{h}])=\int_{\mathbf{R}}^{\oplus} \mathrm{d} \pi_{t}([A, \mathfrak{h}]) \mathrm{d} t .
$$

Il s'ensuit que $d \pi_{t}([A, \mathfrak{h}])=0$ pour presque tout $t \in \mathbf{R}$.

L'hypothèse de récurrence nous permet de dire que $D_{\pi_{t}}(G)^{H}$ est commutative et pour $A, B \in \mathcal{U}_{\pi}(\mathfrak{g})^{\mathfrak{h}}$,

$$
[\mathrm{d} \pi(A), \mathrm{d} \pi(B)]=\int_{\mathbf{R}}^{\oplus} \mathrm{d} \pi_{t}([A, B]) \mathrm{d} t=0
$$


ainsi, nous avons le résultat.

Cas 2: $\mathfrak{h} \not \subset \mathfrak{g}^{Y}$.

Soit $\mathfrak{h}_{1}=\mathfrak{h} \cap \mathfrak{g}^{Y}$ et $H_{1}=\exp \left(\mathfrak{h}_{1}\right)$. Il est clair que $\mathfrak{h}_{1}$ est un idéal de $\mathfrak{g}^{Y}$. Notons dans ce cas que nous pouvons prendre le supplémentaire $\mathfrak{t}=\mathbf{R} X$ dans $\mathfrak{h}$. En utilisant le théorème du sous-groupe de Mackey, nous avons $\pi_{\mid H} \simeq \operatorname{Ind}_{H_{1}}^{H}\left(\pi_{1 \mid H_{1}}\right)$ où $\pi_{1}$ est la représentation associée à $f_{1}=f_{\mid \mathfrak{g}^{Y}}$. La représentation $\pi_{1 \mid H_{1}}$ est en général pas irréductible et si les multiplicités qui apparaissent dans la décomposition en irréductibles de $\pi_{\mid H}$ sont finies, alors il sera encore le cas pour cette représentation. Il s'ensuit que pour presque tout $t \in \mathbf{R}$ la représentation $\pi_{t \mid H_{1}}$ a encore des multiplicités finies. Maintenant par le même argument que dans le premier cas, on peut facilement montrer:

$$
\mathcal{U}_{\pi}(\mathfrak{g})^{\mathfrak{h}} \subset \bigcap_{t \in \mathbf{R}} \mathcal{U}_{\pi_{t}}\left(\mathfrak{g}^{Y}\right)^{\mathfrak{h}_{1}}
$$

L'hypothèse de récurrence appliquée au triplet $\left(\mathfrak{g}^{Y}, \mathfrak{h}_{1}, \pi_{t}\right)$ nous permet de dire que l'algèbre $D_{\pi_{t}}\left(G^{Y}\right)^{H_{1}}$ est commutative. Par le même chemin que dans le premier cas, nous avons le résultat.

COROLLAIRE 5.6. Supposons que G est nilpotent et H un sous-groupe normal de G. Supposons encore que $\pi_{\mid H}$ se décompose avec des multiplicités finies presque pour tout $\pi \in \hat{G}$. Alors le centralisateur de $\mathfrak{h}$ dans $\mathcal{U}(\mathfrak{g})$ déjà noté $c(\mathfrak{h}, 0)$ avec les notations $d u$ paragraphe 2 est une algèbre abélienne.

Dans un prochain travail, nous allons étudier la conjecture 5.2 et aussi le problème de la commutativité des centralisateurs des sous-algèbres dans l'algèbre enveloppante objet du corollaire 5.6.

\section{EXEMPLES 5.7.}

EXEMPLE 1. Soit $G$ un groupe de Lie résoluble exponentiel et $H$ un sous-groupe analytique de $G$. Soit $\pi \in \hat{G}$. Si $H=\{e\}$, alors $D_{\pi}(G)=D_{\pi}(G)^{\{e\}} \simeq \mathcal{U}(\mathrm{g}) / \operatorname{ker}(\mathrm{d} \pi)$ qui est en général une algèbre non-commutative.

EXEMPLE 2. Soit $\mathfrak{n}=\operatorname{vect}(X, Y, Z)_{\mathbf{R}}$ l'algèbre de Heisenberg, i.e $[X, Y]=Z$. Soient $\lambda$ un réel non-nul, $f_{\lambda}=\lambda Z^{\star}$ et $\pi_{\lambda}$ la représentation de $G$ associée à $f_{\lambda}$. Soit encore $\mathfrak{h}=\operatorname{vect}(Z)_{\mathbf{R}}$. Alors

$$
\pi_{\mid H} \simeq \infty \cdot \chi_{\lambda}
$$

où $\chi_{\lambda}$ est le caractère de $H$ définie par $\chi_{\lambda}(\exp (t Z))=\mathrm{e}^{\mathrm{it} \lambda}$. Ainsi $\mathcal{U}_{\pi}(\mathfrak{g})^{\mathfrak{h}}=\mathcal{U}(\mathfrak{g})$ et $D_{\pi}(G)^{H} \simeq \mathbb{C}[X, Y]$ qui est une algèbre non-commutative. 
EXEMPLE 3. Si on reprend le même exemple avec $\mathfrak{h}=\operatorname{vect}(Z, X)_{\mathbf{R}}$. Alors il est facile de voir que

$$
\pi_{\mid H} \simeq \int_{\mathbf{R}}^{\oplus} \chi_{\lambda, t} \mathrm{~d} t
$$

où $\chi_{\lambda, t}$ est le caractère de $H$ défini par $\chi_{\lambda}(\exp (z Z) \exp (x X))=\mathrm{e}^{i z \lambda} \mathrm{e}^{i x t}$.

Considérons le drapeau d'idéaux $\mathcal{S}$ de g:

$$
\mathcal{S}:\{0\} \subset \mathbf{R} Z \subset \mathbf{R} X \oplus \mathbf{R} Z \subset \mathfrak{g} .
$$

Cela implique que $\mathfrak{h}$ est la polarisation de Vergne de $\mathfrak{g}$ en $f_{\lambda}$ par rapport à $\mathcal{S}$ et que $\operatorname{Ind}_{H}^{G} \chi_{f_{\lambda}}$ est irréductible. Ici, $\mathcal{U}_{\pi}(\mathfrak{g})^{\mathfrak{h}}=\mathcal{U}(\mathfrak{h})$. Ainsi $D_{\pi}(G)^{H} \simeq \mathrm{C}[X]$ qui est une algèbre commutative.

Soit $\mathcal{H}_{\lambda}$ l'espace de la représentation $\pi_{\lambda}$. Donc $\mathcal{H}_{\lambda}^{+\infty}$ est l'espace de Schwartz $\mathcal{S}(\exp (\mathbf{R} Y))$. Pour $\xi \in \mathcal{H}_{\lambda}^{+\infty}$, nous avons que $\mathrm{d} \pi(X) \xi(\exp (t Y))=i \lambda t \xi(\exp (t Y))$, et donc

$$
D_{\pi}(G)^{H} \simeq \mathrm{C}[M(t)]
$$

où $M(t)$ est l'opérateur de multiplication par la variable $t$.

Si on réalise la représentation $\pi_{\lambda}$ à l'aide de la polarisation $\mathfrak{b}=\mathbf{R} Y \oplus \mathbf{R} Z$, alors nous obtenons que

$$
D_{\pi}(G)^{H} \simeq \mathbb{C}\left[\frac{\mathrm{d}}{\mathrm{d} t}\right] .
$$

Ici $\mathrm{d} / \mathrm{d} t$ est l'opérateur de dérivation par rapport à la variable $t$. Evidemment, nous pouvons passer de la forme de $D_{\pi}(G)^{H}$ comme dans (12) à la forme de $D_{\pi}(G)^{H}$ comme dans (13) par une transformée de Fourier partielle qui est en réalité un opérateur d'entrelacement de la désintégration (11).

EXEMPLE 4. Si on reprend le même exemple avec $\mathfrak{h}=\operatorname{vect}(X)_{\mathbf{R}}$ qui n'est pas un idéal de g. Il est clair que $\pi_{\mid H}$ est à multiplicités finies. En plus, $D_{\pi}(G)^{H} \simeq \mathrm{C}[X]$ qui est une algèbre commutative.

\section{Remerciements}

Les auteurs tiennent à remercier le référee. Ses nombreux corrections, remarques et commentaires nous ont permis de mettre au point cet article et de le rendre sous la présente forme.

\section{Bibliographie}

1. Baklouti, A.: Harmonic analysis on invariant differential operators on nilpotent homogeneous spaces, Russian J. Math. Physics 6(2) (1999), 125-136.

2. Baklouti, A. and Fujiwara, H.: Harmonic analysis on some exponential solvable homogeneous spaces, Res. Expos. Math. 25(1) (2002), 127-134.

3. Baklouti, A. and Ludwig, J.: Invariant differential operators on certain nilpotent homogeneous spaces, Monatsh. Math. 134 (2001), 19-37. 
4. Benoist, Y.: Analyse harmonique sur les espaces symétriques nilpotents, J. Funct. Anal. 59 (1984), 211-253.

5. Bernat et coll, P.: Représentations des groupes de Lie résolubles, Dunod, Paris, 1972.

6. Corwin, L. and Greenleaf, F. P.: Representations of Nilpotent Lie Groups and Their Applications. Part I: Basic theory and examples, Cambridge University Press, 1990.

7. Corwin, L. and Greenleaf, F.: Commutativity of invariant differential operators on nilpotent homogeneous spaces with finite multiplicity, Comm. Pure. Appl. Math 45 (1992), 681-748.

8. Corwin, L. and Greenleaf, F.: Spectral decomposition of invariant differential operators on certain nilpotent homogeneous spaces, J. Funct. Anal. 108 (1992), 374-426.

9. Currey, B.: Noncommutativity of certain subquotients in the enveloping algebra of a nilpotent Lie algebra, J. Lie Theory 11 (2001), 355-379.

10. Duflo, M.: Opérateurs différentiels invariants sur un espace symétrique, C.R. Acad. Sci. Paris A 289 (1979), 135-137.

11. Duflo, M.: Open Problems in Representation Theory of Lie Groups, edited by T. Oshima (1986), 1-5.

12. Fujiwara, H.: Sur les restrictions des représentations unitaires des groupes de Lie résolubles exponentiels, Invent. Math. 104 (1991), 647-654.

13. Fujiwara, H.: Représentations monomiales des groupes de Lie nilpotents, Pacific J. Math. 127 (1987), 329-351.

14. Fujiwara, H.: Représentations monomiales des groupes de Lie résolubles exponentiels, Progr. Math. 82 (1990), 61-84.

15. Fujiwara, H.: Sur la conjecture de Corwin-Greenleaf, J. Lie Theory 7 (1997), 121-146.

16. Fujiwara, H.: Analyse harmonique pour certaines représentations induites d'un groupe de Lie nilpotent, J. Math. Soc. Japan 50 (1998), 753-766.

17. Fujiwara et, H. and Yamagami, S.: Certaines représentations monomiales d'un groupe de Lie résoluble exponentiel, Adv. Sci. Pure Math. 14 (1988) 153-190.

18. Fujiwara, H., Lion, G. and Mehdi, S.: On the commutativity of the algebra of invariant differential operators on certain nilpotent homogeneous spaces, Trans. Amer. Math. Soc. 353 (2001), 4203-4217.

19. Greenleaf, F.: Geometry of coadjoint orbits and noncommutativity of invariant differential operators on nilpotent homogeneous spaces, Comm. Pure Appl. Math. 53 (2000), 1203-1221.

20. Grélaud, G.: 'Désintégration des représentations induites des groupes de Lie résolubles exponentiels'. Thèse de troisième cycle, Univ. de Poitiers, 1973.

21. Inoue, J.: Monomial representations of certain exponential Lie groups. J. Funct. Anal. 83 (1989), 121-149.

22. Kirillov, A. A.: Représentations unitaires des groupes de Lie nilpotents, Uspekhi Math. Nauk 17 (1962), 57-110.

23. Lipsman, R.: Orbital parameters for induced and restricted representations. Trans. Amer. Math. Soc. 313 (1989), 433-473.

24. Pedersen, N.: On the infinitesimal kernel of irreducible representations of nilpotent Lie groups. Bull. Soc. Math. France 112 (1984), 423-467.

25. Penney, R.: Abstract Plancherel theorem and a Frobenius reciprocity theorem, J. Funct. Anal. 18 (1975), 177-190.

26. Poulsen, N.: On $C^{\infty}$-vectors and intertwining bilinear forms for representations of Lie groups, J. Funct. Anal. 9 (1972), 87-120.

27. Quint, S. R.: 'Decomposition of induced representations of solvable exponential Lie groups'. Dissertation, Univ. of California, Berkeley 1973.

28. Torossian, C.: Opérateurs différentiels invariants sur les espaces symétriques I, Méthodes des orbites, J. Funct. Anal. 117 (1993), 118-173. 